\title{
Analyse intégrée des rendements du blé tendre et de leurs causes de variation dans un réseau de parcelles d'agriculteurs du Thymerais
}

\author{
P Leterme ${ }^{1 *}, \mathrm{H}$ Manichon ${ }^{2}$, J Roger-Estrade ${ }^{3}$ \\ avec la collaboration technique d'O Buisson 4 \\ 1 INRA, BP 29, F35650 Le Rheu; \\ 2 CIRAD, BP 5035, F34032 Montpellier cedex 1; \\ 3 INA-PG, F78850 Thiverval-Grignon; \\ 4 INRA, BP 57, F49071 Beaucouzé cedex, France
}

(Reçu le 22 février 1994 ; accepté le 20 juillet 1994)

\begin{abstract}
Résumé - Cette note présente une démarche de diagnostic global de la culture du blé pratiquée à l'échelle d'une petite région. Les blés étudiés sont cultivés derrière 2 groupes de précédents (blé ou non-blé) en limons caillouteux ou non en Thymerais (Eure-et-Loir, à $80-100 \mathrm{~km}$ à l'ouest de Paris). On montre que, dans beaucoup de parcelles, le fonctionnement du couvert végétal est déjà perturbé avant la montaison, notamment pour des raisons sanitaires. Durant la montaison, le facteur limitant majeur est l'alimentation hydrique. À cette époque, l'effet du milieu domine l'effet du système de culture qui malgré tout existe essentieliement par sa composante succession de cultures : l'état sanitaire des blés sur blé apparaît fréquemment défavorable. L'effet de la sécheresse persiste lors du remplissage des grains mais est d'autant plus fort qu'il existe d'autres caractères défavorables (état structural du sol, états sanitaires des tiges et racines, verse). On observe que les précédents blé induisent plus de risques d'apparition de caractères défavorables que les précédents non-blé, risques renforcés par les semis tardifs et la culture en terres caillouteuses. En conclusion, on note qu'il n'existe pas de relation stricte entre les rendements et les variables décrivant le système de culture et/ou le milieu. On peut cependant dire que ces dernières conduisent à des niveaux de risque différenciés, dont l'extériorisation dépend du climat, et donc de la date de semis.
\end{abstract}

diagnostic cultural / blé tendre / système de culture / élaboration du rendement / modélisation

Summary - Yield analysis of wheat grown in an on-farm field network in Thymerais (France). This paper presents a methodology of analysing yield variations of wheat grown in on-farm fields. The study takes place in 'Thymerais', a region at about 80-100 km in the west of Paris. The wheats studied were grown after 2 groups of previous crops (wheat or non-wheat). The soils are silty and the fields may be stony. In many fields, we can notice an early reduction of crop growth, before spring stem elongation, mainly for sanitary causes. During the stem elongation, the main limiting factor is water nutrition. At present, the environmental effect is more important than that of cropping systems. Nevertheless, the latter exists mainly by its crop rotation component: the sanitary state of the wheat behind

\footnotetext{
* Correspondance et tirés à part
} 
wheat is often deficient. Drought prevails in summer, during grain filling, with larger consequences when there are other limiting factors (soil structure, stem and root sanitary states, and lodging). We observe that wheat as a precedent induces higher risks of yield limitation, these risks being increased by late sowings and stony fields. In conclusion, we notice that there is not an exact relationship between yields and cropping systems and/or environment characteristics. However, we can say that these induce different levels of risk, whose exteriorisation depends on weather and sowing date.

crop diagnosis / wheat / cropping system / yield elaboration / modelling

\section{INTRODUCTION}

La nécessité d'une analyse intégrée des rendements réalisés en culture de blé provient des inquiétudes formulées par nombre d'agriculteurs concernant la "fertilité» de leurs parcelles conduites en rotations céréalières intensives, et plus globablement la pérennité de leurs systèmes de culture. Des diagnostics thématiques (sanitaire, chimique...) ont alors été mis en œuvre (INRA, 1986) mais se sont heurtés à des problèmes de confusion d'effets et aux nombreuses interactions existant au sein d'un écosystème cultivé. En conséquences, l'Office national interprofessionnel des céréales (ONIC) a initié entre 1984 et 1987 une démarche destinée à intégrer à l'échelle de la parcelle les enseignements tirés de ces études analytiques. Cette étude s'est articulée autour de 3 volets : le premier a consisté à choisir une région d'étude et, en son sein, des situations suffisamment variées pour formuler un diagnostic concernant les rotations céréalières intensives, définies comme ayant plus de 2 tiers de céréales dans la rotation et conduites avec des niveaux élevés d'intrants (Capillon et al, 1985). Le deuxième volet a consisté à tester en stations expérimentales la pertinence et la puissance des méthodes de diagnostic disponibles (Leterme, 1987a). Le troisième volet a consisté à mettre en œuvre en vraie grandeur ces méthodes lors de la campagne 1986/1987 dans les situations préalablement définies : 55 parcelles de blé (cultivées avec 2 variétés: Festival et Fidel) dans une petite région du nord du département d'Eure-et-Loir ; le Thymerais. L'objectif de cette note est de présenter ce troisième volet.

Les principes d'analyse employés peuvent se résumer en 4 points :

i) Mettre en évidence les dysfonctionnements de chaque parcelle de blé en comparant les valeurs observées de certaines caractéristiques du peuplement et des valeurs potentielles de référence de ces mêmes caractéristiques. Ces dernières sont établies par modélisation ou à défaut, col- lectées dans des expérimentations ad hoc (Leterme, 1987b).

ii) Caractériser les états du milieu (climat, composantes physiques, chimiques, biologiques du sol) où poussent les blés étudiés, ainsi que l'état des capteurs (feuilles, racines).

iii) Établir les relations entre ces 2 ensembles de données, pour définir les états du milieu ou des capteurs responsables des dysfonctionnements observés.

iv) Enfin, chercher les liaisons entre les états s'avérant défavorables (donc à éviter) ou au contraire favorables (à rechercher) et les caractéristiques de systèmes de culture.

Au-delà du cas particulier de l'étude présentée, la méthodologie utilisée se veut exemplaire pour toute action de diagnostic utile au développement.

\section{MATÉRIEL ET MÉTHODES}

\section{Méthodologie de l'analyse des rendements et du diagnostic cultural}

\section{Bases et principes de l'analyse des rendements}

L'organogenèse aboutit à la formation d'un certain nombre d'organes (épis, grains par exemple, qui correspondent aux composantes du rendement) et la croissance à des biomasses. Ces 2 phénomènes sont en étroite interdépendance. Ce sont les variables qui les caractérisent qui seront utilisées selon le principe d'analyse $n^{\circ} 1$ introduit ci-dessus.

\section{Production potentielle de biomasse}

Elle résulte de la transformation du rayonnement solaire intercepté par les feuilles. De nombreux travaux ont montré la relation existant entre croissance en matière sèche et rayonnement solaire (Monteith, 1972, 1994 par exemple). Pendant une période donnée, le niveau de rayonnement incident n'étant généralement pas modifiable par l'agriculteur, l'accroissement de biomasse, que son interception et son absorption permettent, 
peut être qualifié de potentiel, si l'efficience de conversion est maximale (Amir et Sinclair, 1991). En accord avec la littérature (Gosse et al, 1986), nous avons considéré :

$$
M S=21
$$

avec MS, la biomasse aérienne synthétisée en $\mathrm{g} / \mathrm{m}^{2}$ et I, le rayonnement intercepté en $\mathrm{MJ} / \mathrm{m}^{2}$. L'efficience de conversion a été prise égale à $2 \mathrm{~g}$ de $\mathrm{MS} / \mathrm{MJ}$ : cette valeur représente une moyenne sur le cycle de la culture. Nous avons appliqué cette relation du début montaison jusqu'à la récolte sans tenir compte des baisses d'efficience d'interception du rayonnement de fin de cycle liées à la sénescence des feuilles. De même, nous n'avons pas tenu compte du défaut éventuel de croissance au début de la montaison qui peut conduire à une efficience d'interception limitée. Au début montaison, les biomasses observées varient de 50 à 200 $\mathrm{g} \bullet \mathrm{m}^{-2}$, ce qui correspond à des indices foliaires de 1 à 4 (Meynard, 1985). On peut associer à ces valeurs des efficiences d'interception du rayonnement de l'ordre de 40 à $80 \%$ en appliquant le modèle de Monsi et Saeki (Szeicz, 1974) et compte tenu d'un coefficient de transmission $\mathrm{K}$ du rayonnement au sein du couvert de blé estimé à 0,45 par Thorne et al (1988). II faut par ailleurs considérer que la biomasse varie très rapidement à cette époque (multipliée par 1,8 et 2,5 en respectivement 80 et 140 degrés-jour selon Masle (1980)). Enfin, on observe généralement une relation négative entre indice foliaire et efficience de conversion du rayonnement intercepté (Norman et Arkebauer, 1991) : à faible indice foliaire, l'erreur faite en considérant une efficience d'interception égale à 1 sera donc partiellement compensée par le fait de ne pas augmenter l'efficience de conversion. L'absence de données précises d'indice foliaire et la volonté de déterminer une valeur potentielle, ne souffrant donc pas de sous-estimation, nous ont conduits à adopter une efficience d'interception égale à 1 dès le début montaison pour toutes les parcelles. Ces hypothèses conduisent ainsi à une surestimation de la quantité de rayonnement interceptée par la culture, amenant une surestimation des biomasses potentielles, et donc des écarts aux potentiels, calculés à partir des biomasses réelles.

La quantité de rayonnement solaire incident entre la floraison et la maturité est particulièrement importante : elle nous indique le rendement maximal que l'on peut atteindre, sous l'hypothèse que l'essentiel de l'accumulation de biomasse post-floraison, correspondant au remplissage des grains, résulte de la photosynthèse réalisée au même moment (O'Leary et al, 1985 ; Groot et Spiertz, 1990).

Compte tenu des hypothèses sur les valeurs d'efficience d'interception et de conversion que nous avons utilisées, les niveaux de biomasse potentiels calculés sont certainement surestimés. Nous faisons l'hypothèse que ce biais est relativement peu variable d'une situation à l'autre, sans que nous ayons la possibilité de le vérifier. Ce défaut de paramétrage constitue une limite actuelle de la démarche.

\section{Composantes du rendement}

Le cycle de la culture est constitué d'étapes dont le fonctionnement est sanctionné par la formation de nouveaux organes, pouvant s'interpréter chacun comme un niveau de ramification du précédent organe. On a ainsi :

- le nombre de pieds/ $\mathrm{m}^{2}$ (NP) ;

- le nombre d'épi/pied (NEPP), premier niveau de ramification ;

- le nombre de grains/épi (NGPE), deuxième niveau de ramification ;

- la dernière composante a un statut particulier : c'est le poids individuel du grain (PM).

Le produit de ces 4 composantes est égal au rendement. Le produit des 3 premières est égal au nombre de grains par $\mathrm{m}^{2}$ (NG).

Le niveau atteint par une composante va résulter de la qualité du fonctionnement du peuplement pendant la période où elle se détermine et de la valeur de la composante antérieure rapportée à l'unité de surface, c'est-à-dire exprimée à l'échelle où se réalise l'exploitation du milieu par le peuplement. En effet, même si les phases d'élaboration des composantes successives ne sont pas complètement disjointes, c'est en termes de compétition pour les ressources du milieu qu'il faut mener l'analyse de leurs niveaux. Dans un milieu donné, lorsque la compensation est parfaite, on a une relation hyperbolique entre les valeurs des composantes successives, quand on exprime la première par unité de surface $(A)$ et la seconde $(B)$ par unité de A (fig 1). Par exemple, A sera le nombre de grains par $\mathrm{m}^{2}$ et $B$, le poids par grain. Le produit $C(=A X B)$ correspondra ici au poids de grain par $\mathrm{m}^{2}$, c'est-à-dire au rendement. L'hyperbole est le lieu des points qui correspondent à un produit constant. Sa position, c'est-àdire la valeur de $\mathrm{C}$, dépend des ressources offertes par le milieu et des possibilités d'utilisation de ces ressources par le végétal grâce à ses capteurs (feuilles, racines). Le schéma est complété par le fait que chaque composante de type $B$ présente une valeur maximale $B_{x}$ indépassable, liée au génotype. La production constante de $C$ ne peut être atteinte que si $A$ dépasse un seuil noté $A_{0}$; sous ce seuil, $C$ est limité

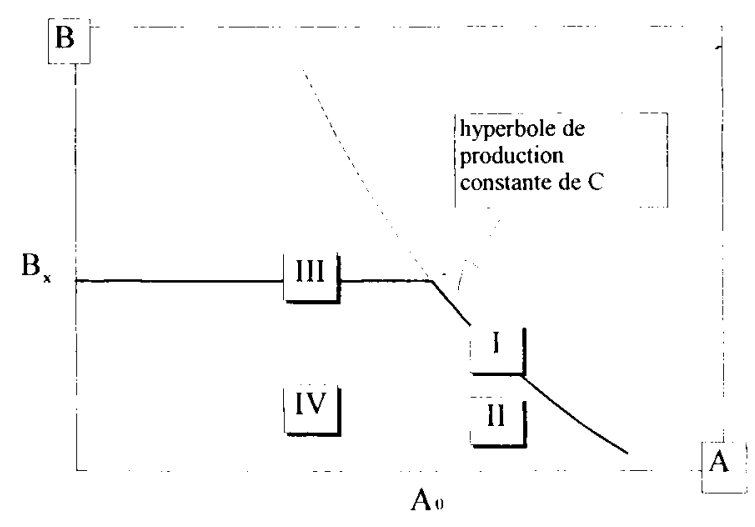

Fig 1. Principe d'analyse de la relation entre 2 composantes successives $A$ et $B$ (par exemple NG et PM). 
car la valeur de $B$ est bornée supérieurement par $B_{x}$. Nous avons représenté, sur la figure 1, 4 positions fictives de parcelles : I, II, III, IV.

- En I, la parcelle réalise la production de $C$ maximale permise par le milieu : toute augmentation d'A se serait traduite par une diminution de $B$. L'effectif minimal d'A permettant d'atteindre ce maximum est $A_{0}$, seuil au-delà duquel apparaît une compensation complète entre composantes.

- En II, la parcelle n'atteint pas le maximum de $C$ bien que la valeur d'A soit suffisante $\left(A>A_{0}\right)$. La limitation doit être recherchée parmi les conditions qui régnaient lors de l'élaboration de $B$.

- En III, le maximum de $C$ n'est toujours pas atteint, mais c'est à cause d'un effectif de $A$ limitant $\left(<A_{0}\right) ; B$ est, quant à lui, au maximum possible $B_{x}$. C'est donc dans les phases antérieures à l'élaboration de $\mathrm{B}$ qu'il faut rechercher l'origine de la limitation.

- En IV, $C$ est très limité, à cause des 2 composantes successives : $A$ est inférieur à $A_{0}$ et $B$ est inférieur à $B_{x}$; des dysfonctionnements se sont manifestés lors des phases d'élaboration des 2 composantes.

Ainsi, lors du remplissage des grains aboutissant finalement au rendement, les ressources maximales seront au moins égales aux produits de la photosynthèse résultant d'une utilisation totale du rayonnement entre la floraison et la maturité. Nous situant par définition au potentiel, les réserves carbonées n'interviendront que peu (Groot et Spiertz, 1990) et la sénescence des feuilles sera très tardive et n'induira pas de baisse importante de l'efficience d'interception. L'efficience de conversion sera maintenue à $2 \mathrm{~g}$ de MS/MJ, la composition biochimique des grains de blé n'impliquant pas une baisse sensible de cette efficience comme ce serait le cas, par exemple, pour les oléagineux à haut contenu énergétique ; cette baisse éventuelle serait par ailleurs plus ou moins compensée par le fait que la croissance est exclusivement aérienne à cette époque, et qu'il n'y a donc plus d'affectation de matière aux racines (Gosse et al, 1986). Ce calcul nous permet ainsi de fixer une estimation du rendement maximal qu'il est possible d'atteindre, compte tenu du climat radiatif et des dates de réalisation des stades.

Si le rendement réel est inférieur au potentiel, on en déduira qu'il a été limité par les composantes NG et/ou $P M$ que l'on analysera selon la figure 1 ( $A=N G, B=$ $P M, C=$ rendement). Le niveau de chaque composante pourra être ainsi déclaré limitant ou non. Ensuite NG sera jugé en référence à la matière sèche végétative accumulée durant la montaison et au nombre d'épis par $\mathrm{m}^{2}$ (NE). Puis seront analysés successivement NE en référence au niveau de biomasse accumulée au début de la montaison (BA1), et $B A 1$ en référence au nombre de pieds par $m^{2}$ (NP) et à la date de semis. Les principes utilisés pour NE et BA1 seront alors présentés.

\section{Établissement de diagnostics sur les écarts aux potentiels}

La figure 2 schématise le déroulement de la
démarche.

Rendement réalisé

Comme nous l'avons signalé plus haut, et sous les hypothèses formulées, nous avons déterminé le rendement en grain potentiel $(R p)$ en considérant que tout le rayonnement incident entre la floraison et la maturité était absorbé et converti en biomasse accumulée dans les grains. En conditions optimales, la durée de fonctionnement a été évaluée à 725 degrés-jour (Gate et

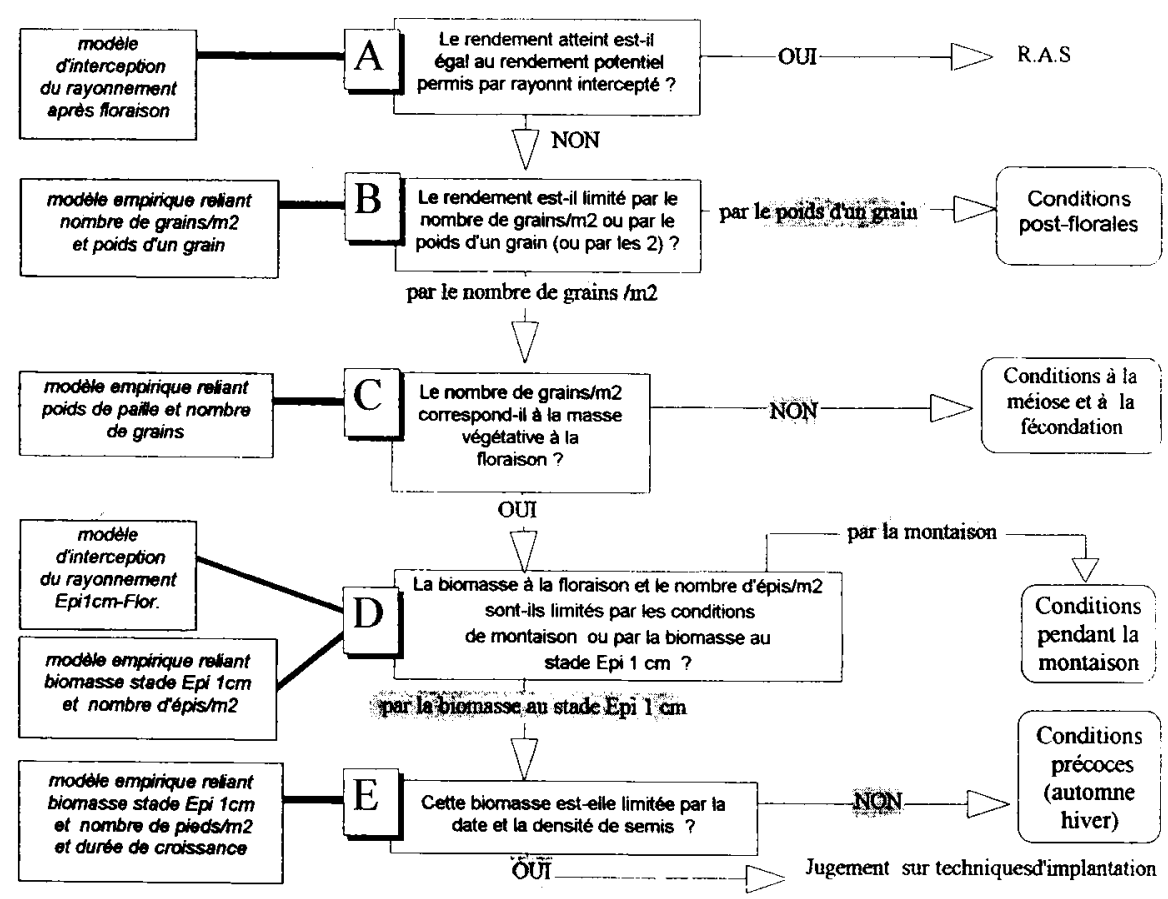

Fig 2. Déroulement du diagnostic. 
Masse, 1988), valeur stable avec la date de floraison et la variété (étape A de la figure 2).

Quand il y avait un écart entre rendements potentiel et réalisé (Rr), nous avons étudié les relations entre le poids d'un grain et le nombre de grains par $\mathrm{m}^{2}$ (NG) de la manière présentée plus haut. Le défaut de rendement imputable à un défaut de remplissage des grains a été estimé par le rapport (noté DeltaR) entre le rendement réalisé et le rendement que l'on aurait obtenu avec le même nombre de grains et le poids individuel de grain correspondant à l'hyperbole de production maximale (cas II de la figure 1) ou celui correspondant au poids de mille grains maximal de la variété (cas IV de la figure 1). Ce défaut de rendement a été mis en relation avec les états du milieu et de la végétation qui régnaient après la floraison : conditions d'alimentation hydrique, états structuraux du sol, état sanitaire des racines, des tiges, verse (étape B). Le défaut de rendement non imputable au remplissage des grains est donc dû à un nombre de grains insuffisant. Le diagnostic sur les causes de cette insuffisance fait l'objet des parties suivantes.

\section{Nombre de grains $/ \mathrm{m}^{2}$}

Le nombre de grains potentiel est étroitement corrélé à la matière sèche végétative élaborée à la floraison (Masle, 1986 ; Meynard et Sebillotte, 1994). Celle-ci est proche du poids de paille, balles et rachis mesurable à la récolte (Masle, 1980 ; Pluchard, 1989). Nous avons donc utilisé ces poids pour estimer dans chaque parcelle le nombre de grains potentiel, en utilisant des relations variétales déterminées antérieurement ou, à défaut, dans un dispositif expérimental qui sera présenté plus bas.

Si le nombre de grains $/ \mathrm{m}^{2}$ est inférieur au potentiel, il peut être limité par les phénomènes perturbant la fertilité de l'épi : l'organogenèse florale peut avoir été défectueuse, mais nous avons privilégié les problèmes pouvant intervenir à la méiose ou à la fécondation (Masle, 1986). Deux critères ont donc été examinés : la température minimale le jour de la méiose (180 et $200 \mathrm{dj}$ avant floraison respectivement pour Festival et Fidel d'après Gate et Masse, 1988) et la satisfaction des besoins hydriques dans les $10 \mathrm{j}$ encadrant la méiose et la floraison (étape $\mathrm{C}$ ). Dans la mesure où ces facteurs apparaîtraient reliés aux caractéristiques de la végétation, il y aurait présomption d'effet sans pour autant pouvoir conclure. En effet, tout autre facteur corrélé à ceux pris en compte pourrait être tenu pour responsable. C'est le cas par exemple des bas niveaux de rayonnement au moment de la méiose, dont l'occurrence est confondue avec celle des basses températures (Demotes, thèse en cours).

Si le nombre de grains $/ \mathrm{m}^{2}$ est égal au nombre de grains potentiel permis par la biomasse végétative mais est cependant limitant du rendement, la seule source de limitation à invoquer réside dans le niveau de cette biomasse. Étant constituée par les tiges, balles et rachis des épis, elle est de fait très liée au nombre d'épis par $\mathrm{m}^{2}$. En effet, à l'échelle du $\mathrm{m}^{2}$, les périodes étant les mêmes, les facteurs qui jouent sur la croissance végétative joueront aussi sur l'élabora- tion du nombre d'épis. On peut ajouter par ailleurs que, durant la montaison, les facteurs pénalisant le fonctionnement du peuplement joueront davantage en modifiant le nombre d'épis qu'en modifiant le poids moyen des parties végétatives de chaque épi. On retrouve ici l'expression d'une loi assez générale qui veut que ce soit plus les nombres d'organes reproducteurs que leurs tailles (ou leur qualité) qui soient affectés par une altération des conditions de fonctionnement des peuplements (Leterme, 1985 et Maillet, 1992 sur colza ; Navarro-Garza, 1984 sur maïs par exemple). En dehors de situations extrêmes, c'est ce qui permet de comprendre la stabilité des indices de récolte.

\section{Biomasse végétative à la floraison et nombre d'épis/ $m^{2}$ (NE)}

La biomasse mesurée est comparée au niveau potentiel d'accroissement de MS résultant de l'interception et la conversion totales du rayonnement solaire incident entre le stade "épi $1 \mathrm{~cm}$ » et la floraison, qui s'ajoute à la biomasse déjà existante au stade "épi $1 \mathrm{~cm}$ ". Le nombre d'épis est potentiellement fixé par la biomasse au stade "épi $1 \mathrm{~cm}$ " (BA1) selon des relations variétales, déterminées antérieurement ou dans le dispositif expérimental d'établissement des références (voir plus bas).

Si le nombre d'épis $/ \mathrm{m}^{2}$ est limitant, l'analyse permet de savoir à quel moment du cycle est liée cette limite (étape D) :

- soit pendant la phase de montaison. Le nombre réel d'épis formés est comparé au nombre d'épis potentiel permis par la biomasse au stade «épi $1 \mathrm{~cm}$ ". On considère que le potentiel est atteint quand l'écart est inférieur à 12\% (Meynard, 1989). Le défaut de réalisation de ce potentiel sera principalement mis en rapport avec les conditions d'alimentation hydrique pendant la montaison, les quantités d'azote disponible estimées sur les bases du bilan prévisionnel de l'azote (Meynard, 1987), l'état structural de la couche labourée (Manichon et al, 1988) et l'état sanitaire des racines aux stades "épi $1 \mathrm{~cm}$ " et floraison ;

- soit avant le stade "épi $1 \mathrm{~cm}$ ". Le nombre d'épis peut être limitant parce que BA1 est trop taible.

\section{Biomasse au stade "épi $1 \mathrm{~cm}$ " (Étape E)}

La valeur de BA1 est déterminée principalement par le nombre de pieds $/ \mathrm{m}^{2}$ et la durée entre levée et stade "épi $1 \mathrm{~cm}$ " exprimée en somme de températures (base 0). Les modalités d'implantation de la culture (date et densité de semis) sont donc très importantes. Nous considérerons aussi les états structuraux du sol (Manichon et al, 1988) et les états sanitaires des racines séminales (Huet, 1988).

Notons que suivant cette analyse, le niveau atteint par chaque composante est relié au contexte de son élaboration. Ce n'est pas la comparaison des valeurs absolues observées dans les parcelles qui permet de formuler les jugements parcellaires mais les écarts aux valeurs potentielles que les composantes auraient atteintes s'il n'y avait pas eu de dysfonctionnement. 


\section{Application au réseau de parcelles étudiées}

\section{Zone d'étude}

La zone d'étude représente un quadrilatère d'environ $10 \mathrm{~km}$ d'est en ouest et $15 \mathrm{~km}$ du nord au sud, situé dans le Thymerais à une vingtaine de $\mathrm{km}$ à l'ouest de Dreux. Le réseau de parcelles étudiées, choisies chez des agriculteurs représentatifs de la diversité des systèmes de culture pratiqués dans la région (Capillon et al, 1985), comprend 55 parcelles, cultivées avec 2 variétés : Fidel et Festival. Dans ces sols (limons lessivés ou dégradés sur argile à silex), la texture est limoneuse avec une teneur en argile croissant avec la profondeur. Deux grandes catégories de parcelles peuvent être distinguées à partir de la teneur en éléments grossiers. Ce critère a été retenu pour stratifier le milieu car il implique probablement des comportements de sols différents ; réserve utile, état structural, dynamique hydrique. Par ailleurs, la conduite de la sole de blé en tient compte : la fréquence des monocultures longues est plus grande en sols caillouteux, les parcelles y sont semées soit en premier, soit en dernier. On a ainsi 27 parcelles sans cailloux et 28 parcelles qui sont au contraire très caillouteuses dès la surface. L'enracinement peut être limité par des zones indurées (grisons) situées à des profondeurs variées et qui limitent la circulation verticale de l'eau. Les réserves utiles maximales s'étalent de 40 à $180 \mathrm{~mm}$.

Les blés étudiés, représentatifs des différents cas existant dans la région (Capillon et al, 1985) sont cultivés derrière au moins 2 blés consécutifs (28 parcelles) et derrière pois, tournesol ou colza d'hiver (27 parcelles). Les dates de semis pratiquées s'échelonnent du $1 / 10$ au $7 / 11$, les dates de floraison du $1 / 6$ au $15 / 6$.

Le tableau I présente la répartition des parcelles selon les précédents, terrains (caillouteux ou non), dates de semis et variétés.

\section{Acquisition des données dans les parcelles du réseau}

\section{Caractéristiques du milieu}

\section{Climat}

Les données météorologiques sont collectées dans 3 micro-stations pour les précipitations et les températures, implantées au nord, au milieu et au sud de la zone. Chaque parcelle est “dotée" des données climatiques recueillies dans la station la plus proche. Les données d'ETP et de rayonnement global ont été obtenues à la station de la Météorologie nationale de Trappes (Yvelines), distante d'environ $50 \mathrm{~km}$ de la zone.

\section{Terrains}

Au sein de chaque parcelle, nous avons délimité une zone de terrain homogène (appelée station) d'environ $500 \mathrm{~m}^{2}$ dans laquelle toutes les observations de végétation et de milieu ont été réalisées.

L'état chimique des sols a été évalué par l'analyse d'un échantillon composite constitué à partir de 10 prises réparties dans chaque station.

Des analyses nématologiques ont été effectuées pour rechercher les nématodes à kystes Heterodera avenae (Rivoal et Marzin, 1988). Un échantillon de sol d'environ $2 \mathrm{~kg}$ a été prélevé dans chaque parcelle (réunion de 10 prises de $200 \mathrm{~g}$ prélevées dans la station étudiée). La recherche et le comptage d'Heterodera avenae ont été effectués sur sol séché au laboratoire.

Un profil cultural a été réalisé durant la montaison dans une zone représentative de chaque station selon la méthode décrite par Manichon et Gautronneau (1987). Cinq types de profils ont été constitués à partir de 2 critères structuraux : le degré de fragmentation des bandes de labour et l'importance des tassements créés par le semis. L'observation des enracinements du blé montre que, parmi ces 5 types, 2 apparaissent contraignants : il s'agit de tous les profils présentant des tassements intenses (état interne des mottes de

Tableau I. Répartition des parcelles selon terrains, précédents, dates de semis et variétés.

Terrains caillouteux

\begin{tabular}{cc}
$\begin{array}{c}\text { Précédent } \\
\text { blé }(B)\end{array}$ \\
\hline $\begin{array}{cc}\text { Semis } & \text { Semis } \\
\text { précoce } & \text { tardif }\end{array}$
\end{tabular}

Terrains non-caillouteux

\begin{tabular}{|c|c|c|c|}
\hline \multicolumn{2}{|c|}{$\begin{array}{l}\text { Précédent } \\
\text { blé }(B)\end{array}$} & \multicolumn{2}{|c|}{$\begin{array}{c}\text { Précédent } \\
\text { non-blé (NB) }\end{array}$} \\
\hline $\begin{array}{c}\text { Semis } \\
\text { précoce }\end{array}$ & $\begin{array}{l}\text { Semis } \\
\text { tardif }\end{array}$ & $\begin{array}{l}\text { Semis } \\
\text { précoce }\end{array}$ & $\begin{array}{l}\text { Semis } \\
\text { tardif }\end{array}$ \\
\hline
\end{tabular}

Variété

\begin{tabular}{lllllllll} 
Fidel & 0 & 1 & 0 & 2 & 0 & 0 & 0 & 7 \\
Festival & 7 & 7 & 5 & 6 & 7 & 6 & 4 & 3 \\
\hline
\end{tabular}


type $\Delta)$ sur plus de $50 \%$ du volume de la couche labourée (Manichon et al, 1988).

Des fosses pédologiques ont été creusées dans chaque parcelle après la floraison : elles ont permis de caractériser le sol en profondeur et de mesurer la profondeur maximale d'enracinement. Ces données ont permis d'estimer la réserve utile (RU) dans chaque parcelle. La satisfaction des besoins en eau est évaluée entre le stade "épi $1 \mathrm{~cm}$ " et la maturité à partir du calcul de l'évolution de la réserve en eau du sol $(R)$ selon les équations suivantes où l'indice $i$ représente le jour, $R U$ la réserve maximale, $P$ et $E T P$ respectivement les pluie et évapotranspiration potentielle :

- $R=R U$ le 15/04, compte tenu de l'enracinement et du climat hivernal ;

- si $0<R_{i-1}<=R U: R_{i}=R_{j-1}+P_{i}-E T P_{i}$;

- si $R_{i-1}=0$, alors $R_{i}=P_{i}-\inf \left(P_{j}, E T P_{i}\right), R_{i}$ étant borné inférieurement par 0 et supérieurement par $R U$.

L'indice de satisfaction hydrique (ISH) est estimé pour le jour i par :

-1 si $R_{i}>0$;

$-\left[\inf \left(P_{i}, E T P_{i}\right)\right] / E T P_{i}$ si $R_{j-1}=0$.

Les valeurs journalières sont sommées sur la période considérée pour calculer l'/SH moyen de la période.

L'azote disponible est estimé en appliquant les références utilisées pour l'application de la méthode des bilans prévisionnels (Taureau, 1987).

\section{Caractéristiques de la végétation}

\section{Rendement et composantes du rendement}

Deux sous-stations d'environ $250 \mathrm{~m}^{2}$ ont été mises en place dès l'automne dans chaque station. L'une est mise en réserve pour la récolte et l'autre reçoit un réseau de 24 placettes ( 2 rangs contigus sur $50 \mathrm{~cm}$ de long) sur lesquelles nous avons compté le nombre de pieds pour évaluer le peuplement.

Douze placettes (tirées au sort dans les 24) ont été prélevées aux environs du stade «épi $1 \mathrm{~cm}$ » pour évaluer la biomasse sèche par $\mathrm{m}^{2}$. Une correction faite à partir de la longueur réelle de la tige et de l'épi permet d'évaluer la biomasse au stade "épi $1 \mathrm{~cm}$ ". D'après Meynard (1985), lorsque la longueur «tige + épi» est comprise entre 8 et $18 \mathrm{~mm}$, la biomasse est proportionnelle à cette longueur. Une simple règle de 3 permet ainsi de corriger les biomasses mesurées pour estimer la biomasse au stade "épi $1 \mathrm{~cm}$ ". On estime par ailleurs la date de réalisation du stade "épi $1 \mathrm{~cm}$ " dans chaque parcelle à partir de la relation observée, au niveau de l'ensemble des parcelles d'une même variété, entre longueur "tige + épi" et somme de température (base 0) depuis le semis. II faut noter que les corrections qui ont été ainsi faites sont toujours restées limitées.

Les 12 autres placettes sont prélevées à maturité pour mesurer le poids sec de grains, le poids sec de balles et pailles, le poids de 1000 grains et le nombre d'épis.

Le rendement est mesuré directement par la pesée du grain récolté lors du passage de la moissonneuse- batteuse de l'agriculteur dans la première sous-station sur une longueur de $50 \mathrm{~m}$ balisée par nos soins. Cela représente une surface homogène de 180 à $210 \mathrm{~m}^{2}$ selon la machine.

Ces données permettent de calculer l'indice de récolte et le nombre de grains par $\mathrm{m}^{2}$.

\section{Observations complémentaires}

Les tailles herbacées présentes aux environs du stade "épi $1 \mathrm{~cm}$ " ont été observées sur 50 plantes par parcelle, prélevées au hasard en dehors des placettes (10 séquences de 5 plantes). La confrontation entre les rangs des feuilles et les talles présentes permet de mettre en évidence et de dater d'éventuels arrêts du tallage herbacé. Compte tenu du niveau de BA1 observé et de la date d'arrêt du tallage, on peut estimer si cet arrêt du tallage s'est réalisé alors que la biomasse dépassait $50 \mathrm{~g} / \mathrm{m}^{2}$ - auquel cas on le qualifie de normal - ou non, ce qui signifie un arrêt prématuré. On a alors un symptôme de dysfonctionnement. Sur ces plantes ont été réalisées les mesures de longueur "tige + épi» utilisées pour préciser la date de réalisation du stade «épi $1 \mathrm{~cm}$.

L'état sanitaire des racines a été observé à 2 époques (Huet, 1988) : au stade “épi $1 \mathrm{~cm}$ " et 10 à 24 j après la floraison. Des prélèvements à la bêche de 5 plantes contiguës ont été effectués à ces 2 dates dans chaque parcelle en 10 endroits. Chaque échantillon est ensuite congelé. Les observations suivantes sont faites au niveau de chacune des plantes.

Au 1 er prélèvement :

- nécroses et anomalies morphologiques en distinguant les systèmes séminal et adventif ;

- état sanitaire : piétin-verse, fusarioses, rhizoctone, mouche grise.

Au $2^{\mathrm{e}}$ prélèvement :

- nécroses et anomalies morphologiques en distinguant les systèmes adventif et d'ancrage ;

- identification et importance des maladies de la base des tiges.

La verse est estimée pour chaque placette prélevée à la récolte par l'évaluation visuelle du pourcentage de tiges couchées à plus de $45^{\circ}$.

Outre la date de réalisation du stade «épi $1 \mathrm{~cm}$ ", nous avons daté la floraison en estimant qualitativement le nombre de tiges fleuries (étamines déhiscentes) lors de passages dans les stations tous les $2 \mathrm{j}$. Par interpolation entre 2 dates successives nous avons estimé le jour où la moitié des épis étaient fleuris ; la précision sur la datation est de l'ordre de la journée. Les dates de méiose et de maturité sont estimées en appliquant des écarts de somme de température par rapport à la date de floraison tirés de la bibliographie (Gate et Masse, 1988).

\section{Dispositif expérimental complémentaire}

Afin de paramétrer les relations variétales potentielles qui nous sont nécessaires comme références pour le diagnostic, nous avons mis en place à Grignon 
(Yvelines), à une cinquantaine de $\mathrm{km}$ du réseau de parcelles, une expérimentation avec les 2 variétés Fidel et Festival (Leterme, 1987b). Le dispositif est installé à l'automne 1985 sur un sol limono-argileux (22\% d'argile) reposant sur du loess calcaire apparaissant vers $70 \mathrm{~cm}$; la réserve utile de ces sols est d'environ $150 \mathrm{~mm}$.

Trois dates et 3 densités de semis ont été réalisées pour la variété Festival. Fidel, variété pour laquelle on disposait déjà des relations de référence nécessaires (Meynard, 1985 ; Leterme, 1987a), a été semée à une seule densité. Quand les relations observées avec Fidel correspondent aux relations de référence définies antérieurement, nous avons fait l'hypothèse que celles observées avec Festival pouvaient nous servir de référence pour cette variété. Le tableau II présente les niveaux des facteurs. Seules les 2 dates de semis les plus précoces $(03 / 10$ et 18/11) ont été utilisées afin d'encadrer celles pratiquées dans le réseau d'enquête. Malheureusement, il n'a pas été possible de semer suffisamment tôt (fin septembre), si bien que cet encadrement n'est pas complet, d'autant plus que la levée a été particulièrement longue et hétérogène dans le dispositif suite à la sécheresse de l'automne 1985. Les références concernant le fonctionnement du peuplement à l'automne ne correspondent donc pas parfaitement à un fonctionnement potentiel. Durant la montaison, en revanche, les conditions de l'essai ont été satisfaisantes et permettent de considérer les relations observées entre BA1 et NE, MSV et NG comme représentant des relations potentielles.

Les références concernant les relations suivantes sont ainsi établies :

- Nombre de pieds par $\mathrm{m}^{2}$ et biomasse au stade «épi $1 \mathrm{~cm}$ " (fig 3). Les points présentés correspondent aux placettes de mesure dans la parcelle ( 2 rangs contigus sur 0,50 de long). Nous avons considéré comme références les droites enveloppant les nuages de points correspondant à chaque date de semis $(03 / 10$ et 18/11) sans que l'on puisse faire de distinction entre Festival et Fidel : nous avons donc considéré une référence unique par période de semis. Les réponses maximales observées dans nos parcelles de Fidel ne sont pas très différentes des réponses potentielles observées antérieurement (Meynard, 1982 ; Leterme, 1987a). Cela dit, il est certain que l'on est confronté à une imprécision qui pousse à la prudence quant aux conclusions.

- Biomasse au stade "épi $1 \mathrm{~cm}$ " et nombre d'épis par $\mathrm{m}^{2}$ (fig 4). La courbe tracée pour Festival correspond à l'ajustement du nuage. Nous avons procédé ainsi par souci d'homogénéité avec les approches antérieures : Meynard (1985) propose la courbe de référence pour la relation entre $\mathrm{BA} 1$ et $\mathrm{NE} / \mathrm{m}^{2}$ présentée à la figure 4 . On constate que les valeurs observées dans notre dispositif sont en accord avec cette relation, ce qui nous permet de dire que le dispositif est apte à fournir la relation de référence pour Festival, que l'on calcule de la même façon. Cette relation de référence pour Festival a pu être utilisée de façon satisfaisante comme enveloppe dans une enquête en parcelles d'agriculteurs conduite dans le Boischaut nord de I'Indre (Lamballe et Sebill, 1986).

- Nombre de grains par $\mathrm{m}^{2}$ et matière sèche végétative à maturité (fig 5).

Ces références doivent être utilisées avec précautions, compte tenu d'une part de l'imprécision inhérente à la détermination empirique des courbes enveloppe et d'autre part de leur acquisition dans le cadre d'une expérimentation conduite une seule année. II conviendra donc d'être prudent dans leur utilisation.

\section{RÉSULTATS ET DISCUSSION}

On a récapitulé au tableau III les résultats observés dans chaque parcelle ainsi que les principaux caractères défavorables diagnostiqués.

\section{Analyse du rendement et de ses composantes}

\section{Relations entre le rendement et ses composantes}

Le rendement est très étroitement lié au nombre de grains $/ \mathrm{m}^{2}$ (fig $6, r=0,94, n=55$ ). On retrouve ici les observations d'autres auteurs (Boiffin et al, 1976 ; Meynard, 1985 ; Sebillotte, 1980 entre

Tableau II. Traitements expérimentaux (Grignon).

\begin{tabular}{lccccc} 
Dates de semis & \multicolumn{3}{c}{ Festival } & & Fidel \\
\cline { 3 - 5 } & & Densité 1 & Densité 2 & Densité 3 & Densité 3 \\
\hline & & & & & \\
$03 / 10$ & fin 10 & 106 & 232 & 363 & 320 \\
$18 / 11$ & $06 / 12$ & 116 & 250 & 334 & 311 \\
$13 / 12$ & $16 / 01$ & 112 & 226 & 366 & 308
\end{tabular}


Fig 3. Établissement des relations de référence entre nombre de pieds et $\mathrm{BA} 1 / \mathrm{m}^{2}$ (Grignon).
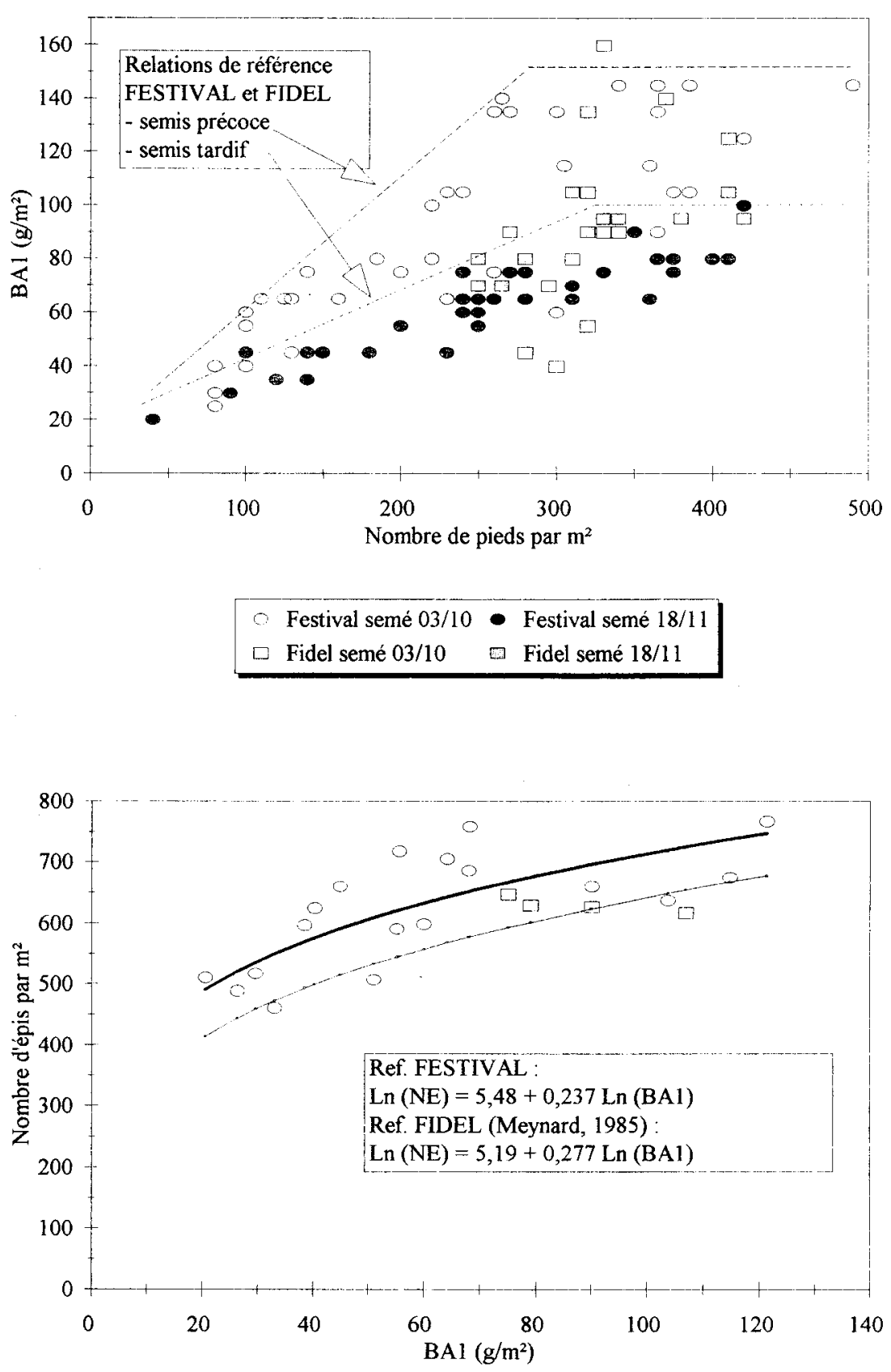

Fig 4. Établissement des relations de référence entre BA1 et nombre d'épis/m² (Grignon).

$\begin{array}{ll}\text { Festival semé } 03 / 10 & \bullet \text { Festival semé } 18 / 11 \\ \square \text { Fidel semé } 03 / 10 & \text { Fidel semé } 18 / 11\end{array}$

autres). Les rendements réalisés vont de 31,4 à $83,9 \mathrm{q} / \mathrm{ha}$ et les nombres de grains de 8110 à 19290 par $\mathrm{m}^{2}$, la relation entre ces 2 variables étant apparemment la même pour les 2 variétés. Les parcelles en terrain caillouteux présentent généralement des nombres de grains plus faibles et surtout plus variables. Le rendement est, en revanche, mal relié au poids individuel des grains $(r=0,22)$ qui est toujours inférieur au potentiel variétal $(P M=55 \mathrm{~g}$ pour Fidel [Meynard, 1985; Leterme, 1987a] et $50 \mathrm{~g}$ pour Festival [Leterme, 1987b]) sauf pour une parcelle de Festival.

\section{Relations entre composantes}

Quand on considère la relation entre nombre de grains par $\mathrm{m}^{2}$ et poids de 1000 grains (fig 7), on observe qu'il existe des parcelles ayant à la fois des faibles valeurs des 2 variables et d'autres où I'on observe des niveaux opposés. En considérant l'enveloppe du nuage de points, il semble que 16000 à 17000 grains $/ \mathrm{m}^{2}$ soit la limite audelà de laquelle il y a compétition : on ne trouve pas de valeurs de PM fortes au-delà de cette valeur. Ce niveau seuil est conforme à la bibliographie (Catin, 1988). Toutes les parcelles de 


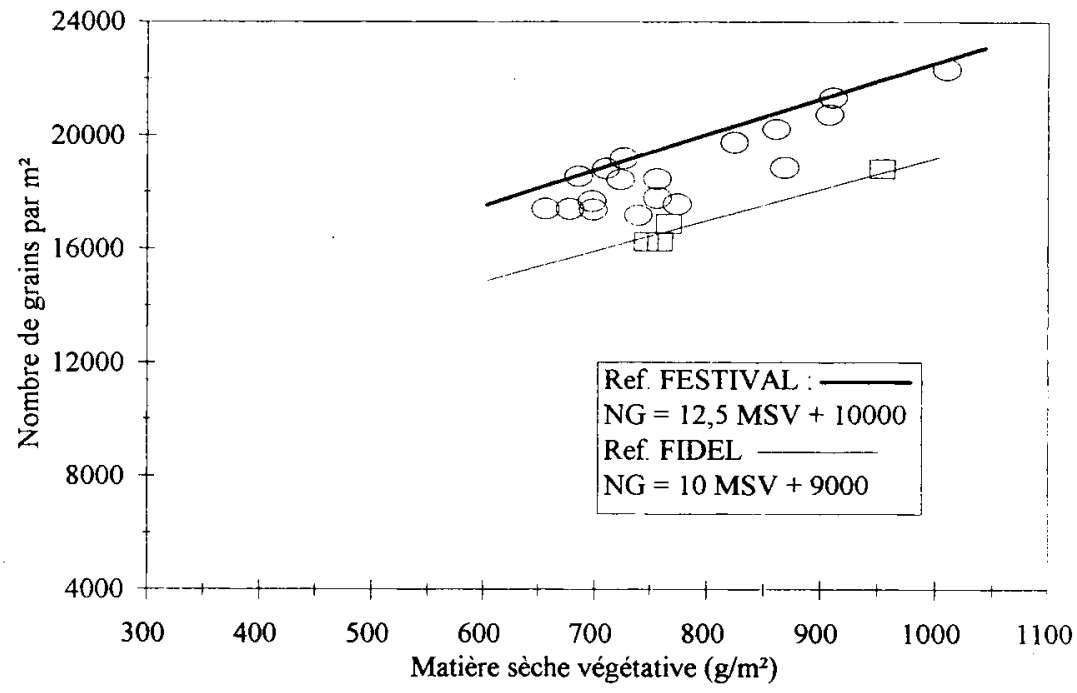

Festival $\square$ Fidel

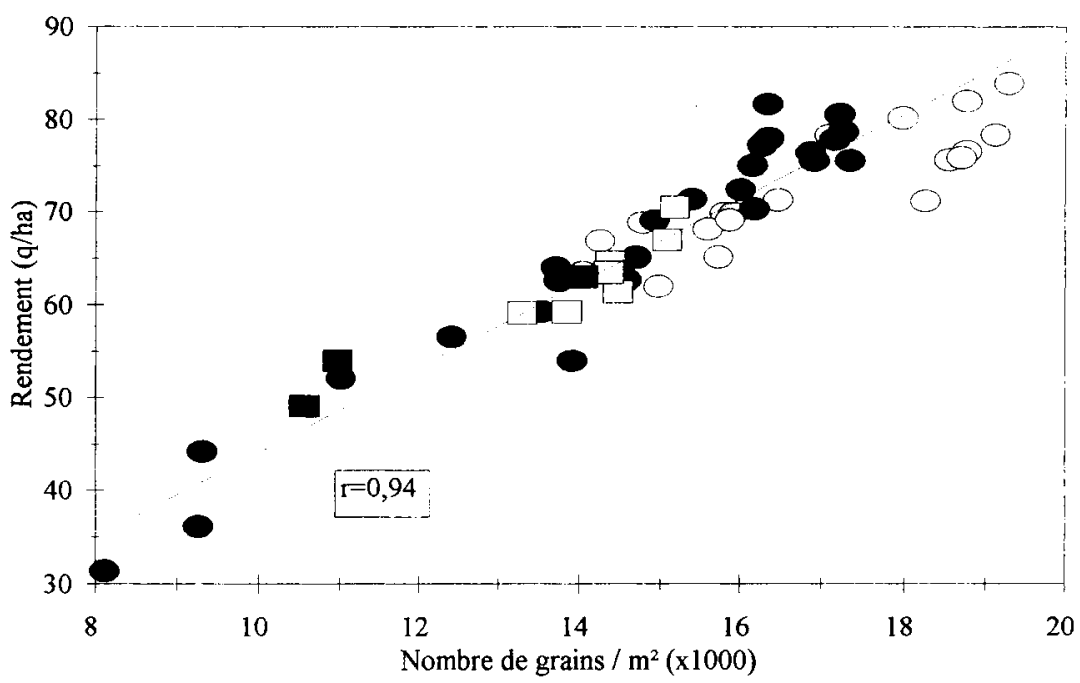

Festival sans cailloux Festival avec cailloux Fidel avec cailloux
Fig 5. Établissement des relations de référence entre matière sèche végétative et nombre de grains $/ \mathrm{m}^{2}$ (Grignon).
Fig 6. Relation entre nombre de grains par $\mathrm{m}^{2}$ et rendement.
Fidel sont sous le seuil, ainsi que majoritairement celles des Festival en terrains caillouteux. Les parcelles de Festival en terrains non caillouteux se répartissent à égalité au-dessus et au-dessous de ce seuil, sans effet des précédents.

\section{Diagnostic sur l'élaboration du rendement et de ses composantes}

Les parcelles sont classées au tableau III par groupes de dates de semis. Cela permet de constituer des ensembles de parcelles ayant des positions de cycles de développement par rapport aux événements climatiques relativement homogènes. Les rendements potentiel et réel sont indiqués ainsi que leur rapport ( $r / p$ ) et les composantes nombre de grains $/ \mathrm{m}^{2}$ et poids de 1000 grains. DI1, DI2, DI3 et DI4 présentent les étapes successives de diagnostic menées dans les parcelles lors de l'élaboration de BA1 (DI1), du nombre d'épis (DI2), du nombre de grains (DI3) et du remplissage de ces grains (DI4). Les principales caractéristiques limitantes du milieu ou du peuplement qui ont été observées sont notées. 
Tableau III. Résultats parcellaires de rendements, composantes et éléments de diagnostic.

\begin{tabular}{|c|c|c|c|c|c|c|c|c|c|c|}
\hline \multirow{2}{*}{$\begin{array}{l}\text { Groupe de } \\
\text { semis }\end{array}$} & \multirow[t]{2}{*}{$\mathrm{N}^{\circ}$} & \multicolumn{2}{|c|}{ Rendements } & \multicolumn{3}{|c|}{$\mathrm{Nb}$ Poids } & \multicolumn{4}{|c|}{ Eléments de diagnostic } \\
\hline & & $\begin{array}{l}\text { (p) } \\
\text { g/ha }\end{array}$ & $\begin{array}{l}\text { (r) } \\
\mathrm{q} / \mathrm{ha}\end{array}$ & $\begin{array}{l}\text { NG } \\
1000 / m^{2}\end{array}$ & PM & $r / p$ & DII & D12 & DI3 & DI4 \\
\hline$I$ & $\overline{03}$ & 95,1 & 76,4 & 16,9 & 45,3 & 0,80 & . & NRH & & \\
\hline \multirow[t]{11}{*}{$(1-7 / 10)$} & 03 & 95,6 & 52,1 & 11,0 & 47,3 & 0,54 & . & $\mathrm{RH}$ & $\mathrm{T}$ & \\
\hline & 02 & 92,7 & 82,0 & 18,8 & 43,7 & 0,88 & & & $\mathrm{~T}$ & IV \\
\hline & 03 & 92,9 & 80,6 & 17,2 & 46,8 & 0,87 & $\mathrm{R}$ & $\mathrm{H}$ & $\mathrm{T}$ & \\
\hline & 01 & 92,9 & 75,7 & 18,6 & 40,8 & 0,81 & . & & $\mathrm{T}$ & I \\
\hline & 04 & 93,4 & 75,1 & 16,2 & 46,5 & 0,80 & & & . & \\
\hline & 01 & 92,1 & 69,9 & 15,8 & 44,2 & 0,76 & & $\mathrm{~N}$ & $\mathrm{~T}$ & HI \\
\hline & 03 & 92,9 & 69,1 & 14,9 & 46,3 & 0,74 & $\mathrm{R}$ & $\mathrm{H}$ & $\mathrm{T}$ & . \\
\hline & 01 & 93,4 & 66,9 & 14,3 & 46,9 & 0,72 & $\mathrm{R}$ & RHI & & \\
\hline & 01 & 92,7 & 65,1 & 15,7 & 41,4 & 0,70 & . & $\mathrm{N}$ & $T$ & HI \\
\hline & 01 & 92,1 & 63,5 & 14,1 & 45,2 & 0,69 & . & $\mathrm{N}$ & $\mathrm{T}$ & I \\
\hline & 04 & 92,9 & 62,7 & 13,8 & 45,6 & 0,67 & & $\mathrm{NH}$ & $T$ & \\
\hline \multirow{11}{*}{$\begin{array}{c}\bar{J} \\
(8-9 / 10)\end{array}$} & $\overline{03}$ & 95,6 & 81,7 & 16,3 & 50,0 & 0,85 & . & $\mathrm{H}$ & $T$ & \\
\hline & 04 & 95,6 & 77,3 & 16,3 & 47,5 & 0,81 & & $\mathrm{H}$ & $\mathrm{T}$ & \\
\hline & 02 & 95,6 & 68,9 & 14,8 & 46,6 & 0,72 & $\mathrm{P}$ & $\mathrm{N}$ & $\mathrm{T}$ & I \\
\hline & 03 & 95,1 & 64,0 & 13,7 & 46,7 & 0,67 & . & $\mathrm{H}$ & & \\
\hline & 01 & 92,1 & 83,9 & 19,3 & 43,5 & 0,91 & . & - & $T$ & \\
\hline & 02 & 92,9 & 78,3 & 17,1 & 45,8 & 0,84 & & . & $\mathrm{T}$ & \\
\hline & 04 & 93,4 & 78,0 & 16,4 & 47,7 & 0,84 & . & & & . \\
\hline & 04 & 92,9 & 72,5 & 16,0 & 45,3 & 0,78 & - & & NT & $I$ \\
\hline & 02 & 92,7 & 69,9 & 15,9 & 43,9 & 0,75 & & $\mathrm{~N}$ & $\mathrm{~T}$ & $\mathrm{H}$ \\
\hline & 01 & 93,4 & 68,2 & 15,6 & 43,7 & 0,73 & $\mathrm{P}$ & . & $N$ & I \\
\hline & 03 & 92,9 & 62,7 & 14,6 & 43,0 & 0,67 & & $\mathrm{NH}$ & $\mathrm{T}$ & I \\
\hline \multirow{22}{*}{$\begin{array}{c}\mathrm{ID} \\
(10-17 / 10)\end{array}$} & 03 & 95,6 & 77,9 & 17,2 & 45,4 & 0,81 & . & $\mathrm{R}$ & $T$ & . \\
\hline & 03 & 95,6 & 56,6 & 12,4 & 45,6 & 0,59 & & $\mathrm{H}$ & $\mathrm{T}$ & I \\
\hline & 14 & 95,6 & 54,0 & 11,0 & 49,2 & 0,56 & & NRH & & IV \\
\hline & 03 & 95,6 & 44,2 & 9,3 & 47,5 & 0,46 & $\mathrm{R}$ & RH & $\mathrm{T}$ & I \\
\hline & 03 & 95,1 & 36,1 & 9,3 & 39,0 & 0,38 & $\mathrm{P}$ & RII & $\mathrm{N}$ & \\
\hline & 04 & 92,1 & 78,7 & 17,3 & 45,6 & 0,85 & & & $\mathrm{HT}$ & $\mathrm{H}$ \\
\hline & 04 & 92,1 & 75,6 & 16,9 & 44,7 & 0,82 & & & NT & \\
\hline & 02 & 92,7 & 71,3 & 16,5 & 43,3 & 0,77 & & & $T$ & $\mathrm{H}$ \\
\hline & 04 & 92,9 & 71,4 & 15,4 & 46,4 & 0,77 & & NH & $\mathrm{T}$ & \\
\hline & 03 & 92,1 & 65,1 & 14,7 & 44,3 & 0,71 & C & & НГ & $\mathrm{HI}$ \\
\hline & 04 & 92,7 & 59,3 & 13,5 & 43,8 & 0,64 & . & $\mathrm{H}$ & NT & 10 \\
\hline & 02 & 91,1 & 80,2 & 18,0 & 44,6 & 0,88 & . & $\mathrm{R}$ & & $\mathrm{H}$ \\
\hline & 04 & 91,1 & 75,6 & 17,3 & 43,6 & 0,83 & . & & $\mathrm{NH}$ & $\mathrm{H}$ \\
\hline & 04 & 91,1 & 70,4 & 16,2 & 43,5 & 0,77 & & & $\mathrm{H}$ & $\mathrm{H}$ \\
\hline & 01 & 90,4 & 69,2 & 15,9 & 43,6 & 0,77 & $\mathrm{R}$ & $\mathrm{R}$ & . & $\mathrm{H}$ \\
\hline & 12 & 91,1 & 64,6 & 14,4 & 44,9 & 0,71 & P & . & . & IV \\
\hline & 02 & 91,4 & 62,8 & 14,5 & 43,2 & 0,69 & $\mathrm{C}$ & . & . & $\mathrm{HI}$ \\
\hline & 14 & 91,1 & 63,1 & 14,1 & 44,9 & 0,69 & $\mathrm{C}$ & $\mathrm{H}$ & $\mathrm{HT}$ & HIV \\
\hline & 03 & 91,1 & 31,4 & 8,1 & 38,7 & 0,34 & $\mathrm{RP}$ & $\mathrm{NRH}$ & $\mathrm{NH}$ & $\mathrm{H}$ \\
\hline & 01 & 87,8 & 78,4 & 19,1 & 41,0 & 0,89 & $\mathrm{C}$ & . & & $\mathrm{HI}$ \\
\hline & 12 & 87,8 & 67,0 & 15,1 & 44,4 & 0,76 & . & . & $\mathrm{N}$ & I \\
\hline & 12 & 88,2 & 61,4 & 14,5 & 42,4 & 0,70 & & & $\mathrm{NH}$ & $\mathrm{H}$ \\
\hline \multirow{10}{*}{$(24 / 10-7 / 11)$} & 13 & 95,1 & 49,1 & 10,6 & 46,5 & 0,52 & $\mathrm{C}$ & $\mathrm{H}$ & $\mathrm{NH}$ & $\mathrm{H}$ \\
\hline & 12 & 92,1 & 59,2 & 13,3 & 44,5 & 0,64 & $\mathrm{P}$ & $\mathrm{R}$ & & IV \\
\hline & 01 & 90,4 & 76,6 & 18,8 & 40,8 & 0,85 & $\mathrm{P}$ & $\mathrm{R}$ & $\mathrm{N}$ & $\mathrm{H}$ \\
\hline & 03 & 90,4 & 54,0 & 13,9 & 38,8 & 0,60 & $\mathrm{P}$ & $\mathrm{H}$ & $\mathrm{H}$ & $\mathrm{H}$ \\
\hline & 01 & 86,3 & 75,9 & 18,7 & 40,6 & 0,88 & $\mathrm{RC}$ & & & $\mathrm{H}$ \\
\hline & 01 & 86,3 & 71,2 & 18,3 & 39,0 & 0,83 & $\mathrm{R}$ & $\mathrm{H}$ & & $\mathrm{H}$ \\
\hline & 12 & 87,8 & 70,6 & 15,2 & 46,5 & 0,80 & & . & . & IV \\
\hline & 12 & 87,4 & 63,5 & 14,4 & 44,2 & 0,73 & $P$ & . & . & HIV \\
\hline & 01 & 87,8 & 62,0 & 15,0 & 41,4 & 0,71 & $\mathrm{R}$ & NR & . & $\mathrm{H}$ \\
\hline & 12 & 88,2 & 59,3 & 13,9 & 42,8 & 0,67 & $\mathrm{P}$ & & & $\mathrm{H}$ \\
\hline
\end{tabular}

Caractères défavorables : : aucun caractère défavorable détecté. $C$ : état chimique ; $N$ : azote limitant ; $P$ : état structural du profil cultural ; $\mathrm{H}$ : stress hydrique durant la montaison (DI2), à la méiose (DI3) après floraison (DI4) ; $R$ : état sanitaire des racines à la montaison (DI1), à la floraison (DI2); $T$ : conditions climatiques à la méiose (température) ; $\mathrm{l}$ : état sanitaire des tiges ; $\mathrm{V}:$ verse.

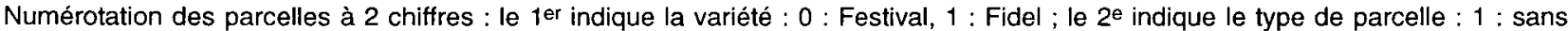
cailloux, précédent blé ; 2 : sans cailloux, non-blé ; 3 : avec cailloux, blé ; 4 : avec cailloux, non blé. 


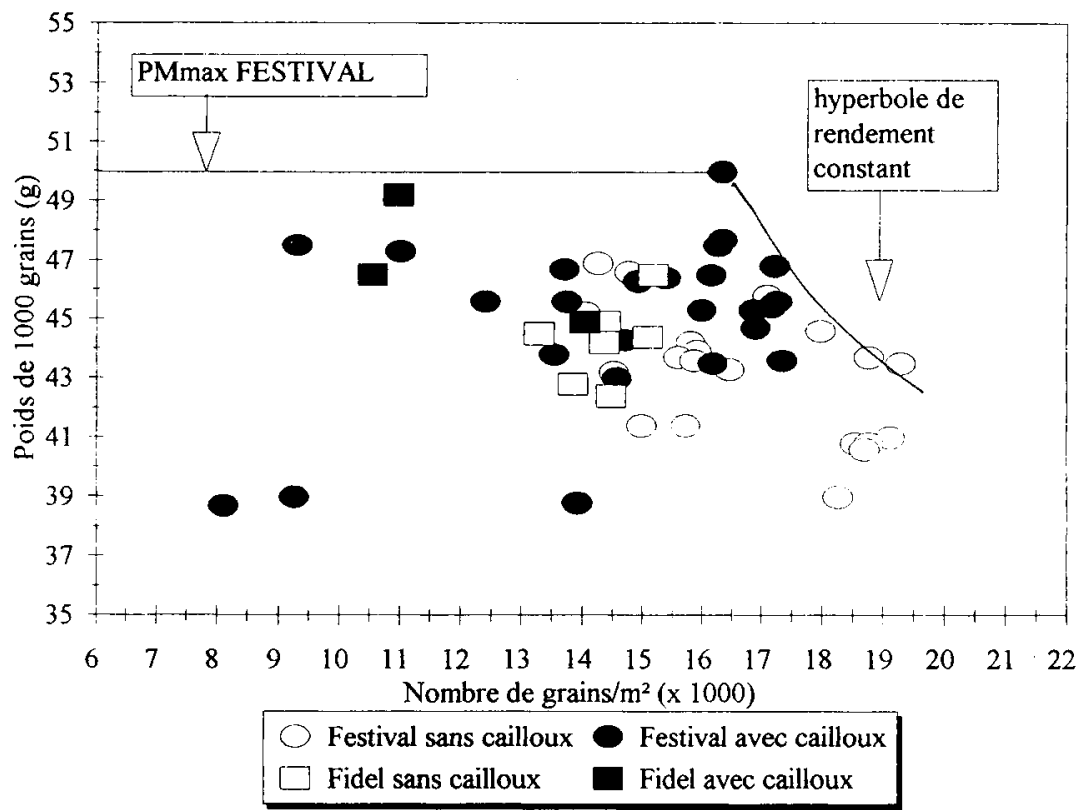

Fig 7. Relation entre poids de 1000 grains et nombre de grains par $\mathrm{m}^{2}$.

\section{Élaboration du nombre d'épis $/ \mathrm{m}^{2}$}

\section{Détermination du potentiel-épi (DI1)}

Pour une variété donnée, il dépend de la biomasse au stade "épi $1 \mathrm{~cm}$ ". Celle-ci est largement influencée par la date de semis (qui entraîne une plus ou moins longue période de croissance entre levée et stade "épi $1 \mathrm{~cm}$ " exprimée en sommes de températures) et par le peuplement. L'expérimentation complémentaire indique que la BA1 maximale en semis précoce (avant le 10/10) est de l'ordre de $150 \mathrm{~g} / \mathrm{m}^{2}$ contre 100 en semis plus tardifs (fig 3 ). Bien que déterminés dans des conditions non optimales, ces ordres de grandeur ont été confirmés par Meynard en 1989. II aurait été souhaitable de disposer d'une variation continue de la référence en fonction de la date de semis mais les contraintes expérimentales ne l'ont pas permis ; faute d'une précision suffisante, nous n'avons pas cherché à calculer par interpolation des valeurs de référence valables pour chaque date de semis et avons préféré un mode de raisonnement qualitatif. La figure 8 montre les relations observées dans les parcelles entre BA1 et NP par groupes de dates de semis. Nous y avons fait figurer les relations de référence tirées de l'expérience complémentaire. Quelle que soit la variété, on observe un assez bon accord bien qu'il existe des parcelles semées très précocement qui dépassent la référence. Rappelons que celle-ci n'a pu être établie pour des semis si précoces et que les conditions étaient particulièrement sèches.

Le tableau IV présente la ventilation des parcelles selon 3 critères :
- la date de semis (précoce, avant le 10/10 ou tardif après le 10/10);

- le fait que la référence de BA1 soit atteinte ou non ;

- l'observation de caractères défavorables qui peuvent être : i) un arrêt du tallage herbacé prématuré (avant que la biomasse accumulée n'atteigne $50 \mathrm{~g} / \mathrm{m}^{2}$ [Masle, 1980]) ; ii) un état sanitaire des racines séminales défavorable (plus de $10 \%$ de longueur nécrosée ou plus de $20 \%$ de racines affectées par des nématodes) ; iii) un état chimique déficient (au moins un macro-élément insuffisant) ; iv) la présence de plus de 5 larves d'Heterodera avenae par $\mathrm{g}$ de terre (Rivoal et Marzin, 1988) ; v) un profil cultural contraignant.

En terrains caillouteux, on atteint rarement (2 cas sur 12 soit $17 \%$ ) la valeur de référence de BA1 correspondant aux semis précoces (150 $\mathrm{g} / \mathrm{m}^{2}$ ), même dans les parcelles où l'on n'identifie aucun caractère défavorable. En revanche, en non-cailloux, elle est plus fréquemment atteinte ( 6 cas sur 11 soit $55 \%$ ) et, quand ce n'est pas le cas, on repère assez facilement le(s) facteur(s) responsable(s).

La référence des semis tardifs étant plus faible $\left(100 \mathrm{~g} / \mathrm{m}^{2}\right)$, on pourrait penser qu'elle est plus facile à atteindre. II n'en est rien car ces semis présentent plus fréquemment des caractères défavorables. Seulement 6 parcelles sur 32 (19\%) apparaissent sans défauts.

Sous réserve que les références utilisées pour évaluer BA1 soient satisfaisantes, on peut dire que beaucoup de parcelles sont déjà perturbées à cette étape du cycle, alors qu'aucun événe- 

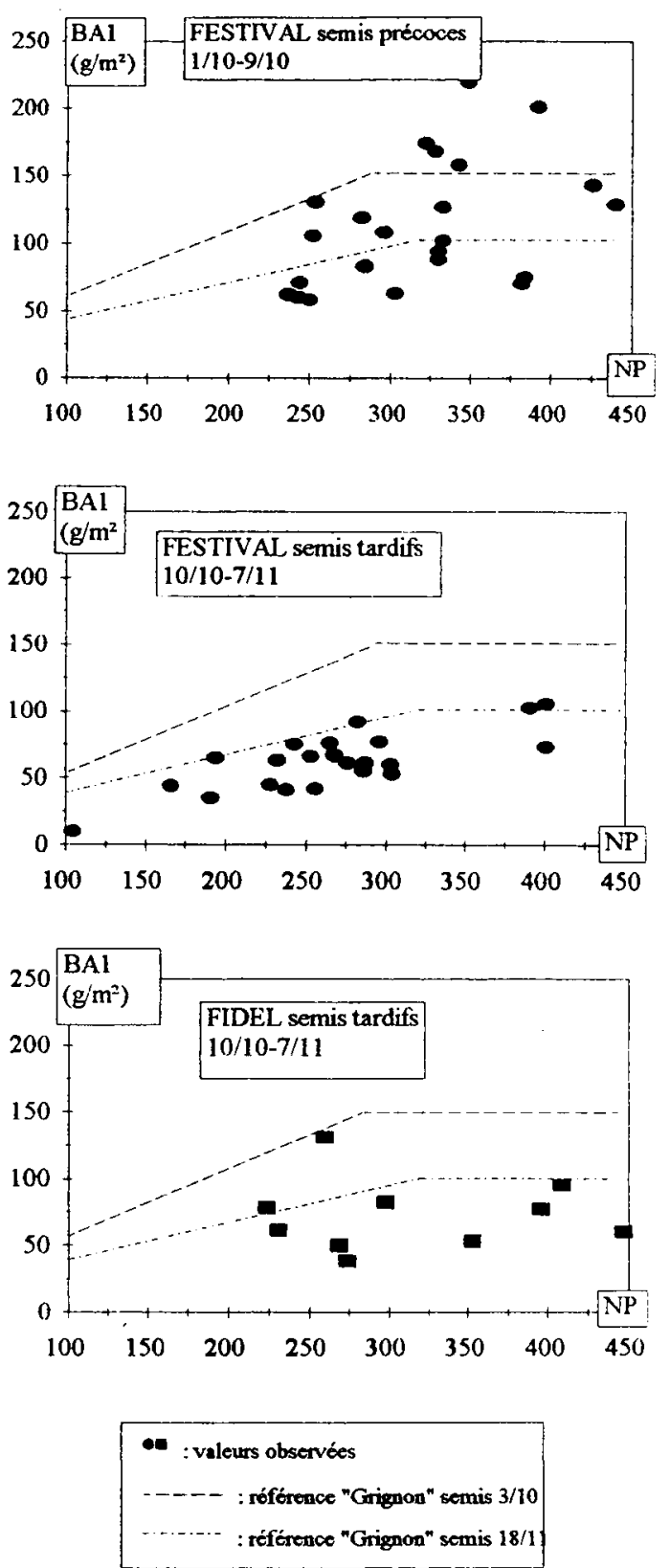

Fig 8. Relations entre NP et BA1.

ment climatique particulier (sécheresse, gel) n'est intervenu. L'examen détaillé de la nature des causes de perturbation met en avant la composante sanitaire et, par là, la succession de cultures : plus de $60 \%$ des parcelles présentent un état sanitaire des racines défavorable en précédent blé, contre seulement $33 \%$ en précédent non-blé, et encore dans ce cas sont-elles faiblement atteintes. On retrouve ici des résultats classiques (Huet, 1988 ; Rivoal et Marzin, 1988).

\section{Réalisation du potentiel-épi (Dl2)}

Pour ne pas surcharger les figures, nous ne représenterons que les parcelles cultivées en
Festival. Nous mentionnerons les comportements particuliers de Fidel quand ils existent. La figure 9 présente la relation entre BA1 et NE ainsi que la courbe de référence décrivant la réponse potentielle (encadrée par $\pm 12 \%$ ).

Nous avons analysé les écarts à ce potentiel en considérant, comme précédemment, l'état sanitaire des racines et l'état structural du profil. Nous avons considéré en plus, compte tenu des besoins de croissance importants à cette époque :

i) la quantité d'azote disponible, estimée à partir du bilan azoté ;

ii) les conditions d'alimentation hydrique pendant la montaison (ISH) ;

iii) l'état sanitaire des racines de tallage, à partir des longueurs nécrosées et des symptômes de lésions par nématodes.

Le tableau $V$ présente la répartition des parcelles selon, d'une part, le niveau du potentiel (approché par la valeur de BA1) et, d'autre part, le degré de réalisation de ce potentiel. Nous avons aussi indiqué les nombres de parcelles affectées par un ISH moyen durant la montaison inférieur à 0,9 , un état sanitaire des racines de tallage au-delà des seuils de nuisibilité déterminés par Huet (1988) et un profil cultural défavorable.

On note qu'il est d'autant plus difficile d'atteindre le potentiel que BA1 est élevé : seulement $12 \%$ des parcelles avec BA1 supérieure à $100 \mathrm{~g} / \mathrm{m}^{2}$ y parviennent, contre $45 \%$ pour les autres. Ainsi la réalisation des forts potentiels exige que toutes les conditions, y compris celles régnant pendant la phase précédente, soient favorables. Inversement, de faibles potentiels peuvent être atteints alors que certaines conditions sont défavorables. Le bas du tableau $V$ résume les informations concernant la nature des causes de dysfonctionnements. C'est l'alimentation hydrique qui ressort comme contrainte majeure : les forts défauts de réalisation des potentiels-épis sont concomitants avec des déficits hydriques dans $88 \%$ des parcelles et l'analyse montre qu'on n'atteint jamais le potentiel-épi dès que I'ISH est inférieur à 0,7 . On observe un effet parallèle de l'état sanitaire des racines, mais qui est moins systématique. L'effet du profil cultural ne ressort pas distinctement.

Ainsi l'effet du milieu, dont dépend largement l'alimentation hydrique, domine l'effet du système de culture. Celui-ci existe malgré tout par sa composante succession culturale. En effet, l'état sanitaire des racines est généralement plus mau- 
Tableau IV. Répartition des écarts à la référence de BA1 selon les types de parcelles.

\begin{tabular}{|c|c|c|c|c|c|c|c|c|c|}
\hline & & \multicolumn{8}{|c|}{ Référence de $B A 1$} \\
\hline & & \multicolumn{4}{|c|}{ Atteinte } & \multicolumn{4}{|c|}{ Non atteinte } \\
\hline & & $\begin{array}{c}B \\
T F\end{array}$ & $\begin{array}{l}N B \\
T F\end{array}$ & $\begin{array}{r}B \\
T C\end{array}$ & $\begin{array}{l}N B \\
T C\end{array}$ & $\begin{array}{c}B \\
T F\end{array}$ & $\begin{array}{l}N B \\
T F\end{array}$ & $\begin{array}{r}B \\
T C\end{array}$ & $\begin{array}{l}N B \\
T C\end{array}$ \\
\hline \multirow[t]{2}{*}{$\begin{array}{l}\text { Semis }|-1| \\
(\leq 9 / 10)\end{array}$} & $\begin{array}{l}\text { Sans conditions } \\
\text { défavorables }\end{array}$ & 4 & 2 & 1 & 1 & 0 & 0 & 1 & 2 \\
\hline & $\begin{array}{l}\text { Avec conditions } \\
\text { défavorables }\end{array}$ & 0 & 0 & 0 & 0 & 3 & 2 & 5 & 2 \\
\hline \multirow[t]{2}{*}{$\begin{array}{l}\text { Semis III-IV } \\
(\geq 10 / 10)\end{array}$} & $\begin{array}{l}\text { Sans conditions } \\
\text { défavorables }\end{array}$ & 0 & 2 & 1 & 1 & 0 & 1 & 0 & 1 \\
\hline & $\begin{array}{l}\text { Avec conditions } \\
\text { défavorables }\end{array}$ & 3 & 0 & 4 & 2 & 3 & 7 & 3 & 4 \\
\hline
\end{tabular}

Précédent B : blé ; NB : non-blé ; terrain : TF : parcelle sans cailloux; TC : parcelle avec cailloux.

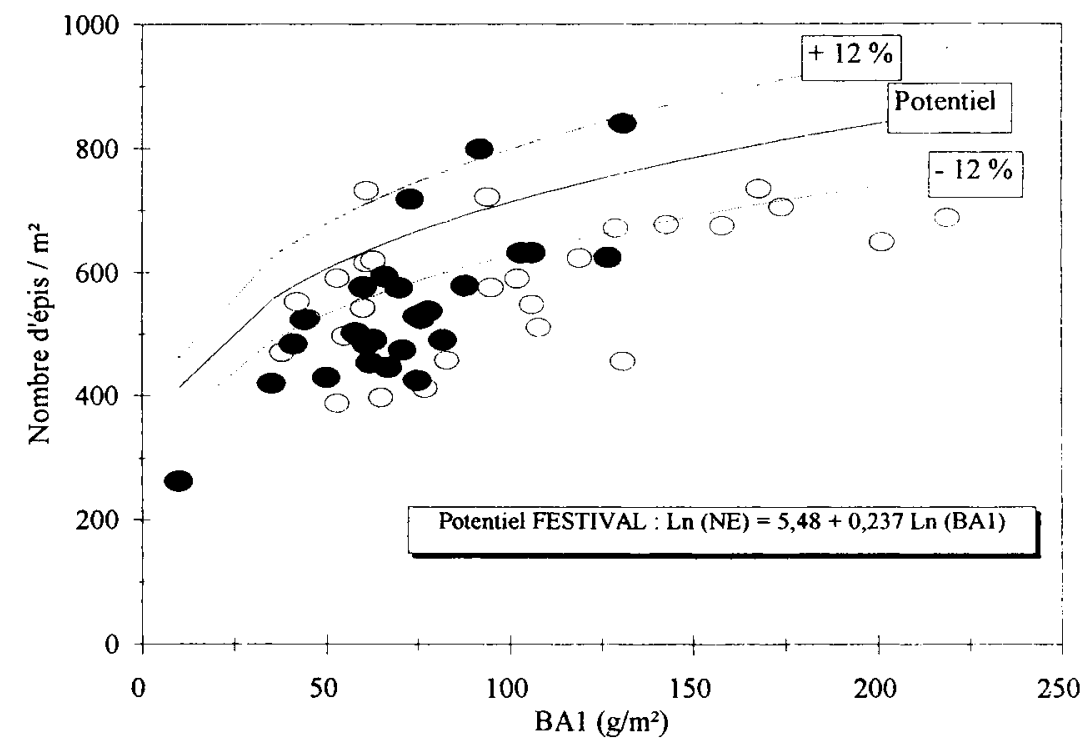

Sans cailloux Cailloux

Fig 9. Relation entre BA1 et nombre d'épis par $\mathrm{m}^{2}$ (Festival). vais en précédent blé comme on peut le constater au tableau VI.

\section{Élaboration du nombre de grains $/ \mathrm{m}^{2}$ (DI3)}

\section{Détermination du potentiel}

Le fonctionnement du peuplement durant la montaison peut s'analyser à partir de la biomasse accumulée, rapportée à la biomasse potentielle permise par le rayonnement incident. Nous avons calculé DeltaM, égal au rapport entre l'ac- croissement de biomasse observé entre «épi 1 $\mathrm{cm}$ » et floraison et l'accroissement potentiel entre ces 2 mêmes stades. La figure 10 montre que ce rapport varie de 0,34 à un peu plus de 1 essentiellement en fonction du degré de satisfaction des besoins en eau durant la montaison. Comme on en avait fait l'hypothèse lors du choix du critère "cailloux", on peut constater que la valeur de ce rapport recoupe la distinction des terrains : en sols caillouteux, les réserves utiles plus faibles et le calendrier des dates de semis différent aboutissent à des ISH fréquemment 
Tableau V. Répartition en nombre de parcelles des modalités de réalisation du peuplement-épi.

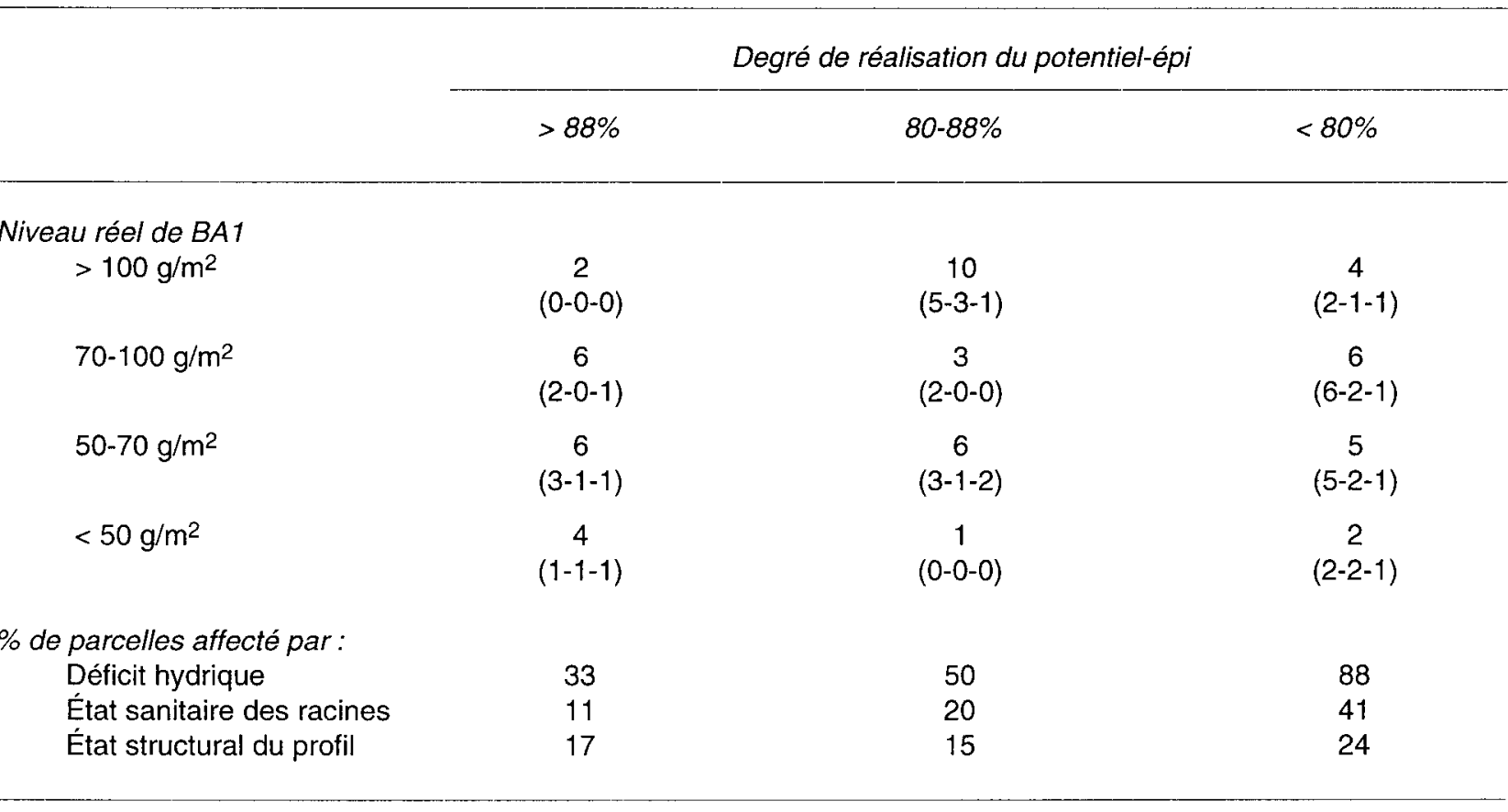

$(x-y-z): x$ : nombre de parcelles avec ETR/ETM moyen durant la montaison $<0,9, y:$ nombre de parcelles avec un état sanitaire des racines adventives défavorable, $z:$ nombre de parcelles avec état structural du profil cultural défavorable.

Tableau VI. Distribution des parcelles selon précédents et états sanitaires des racines adventives.

État sanitaire des racines adventives

$\frac{\text { Précédent }}{\text { Blé Non-blé }}$

a $<2 \%$ d'indice de longueur nécrosée

$<7 \%$ des racines avec lésions de nématodes

$b$

2-3\% d'indice de longueur nécrosée

13

$7-14 \%$ de racines avec lésions de nématodes

c

3-4\% d'indice de longueur nécrosée

$14-21 \%$ de racines avec lésions de nématodes

$d$

$>4 \%$ d'indice de longueur nécrosée

$>21 \%$ des racines avec lésions de nématodes
5

8

D'après Huet (1988), les états $c$ et $d$ entraînent des dysfonctionnements.

plus faibles qu'en terres non caillouteuses. Quand on considère l'enveloppe du nuage de points (marquée par un pointillé sur la figure 10), on constate qu'elle correspond approximativement à un effet proportionnel du degré de satisfaction des besoins en eau. Les parcelles éloi- gnées de l'enveloppe sont caractérisées essentiellement par des états sanitaires des racines et des profils culturaux défavorables. Cela est cohérent avec l'hypothèse que ces caractéristiques entraînent une moins bonne efficience de l'eau. 


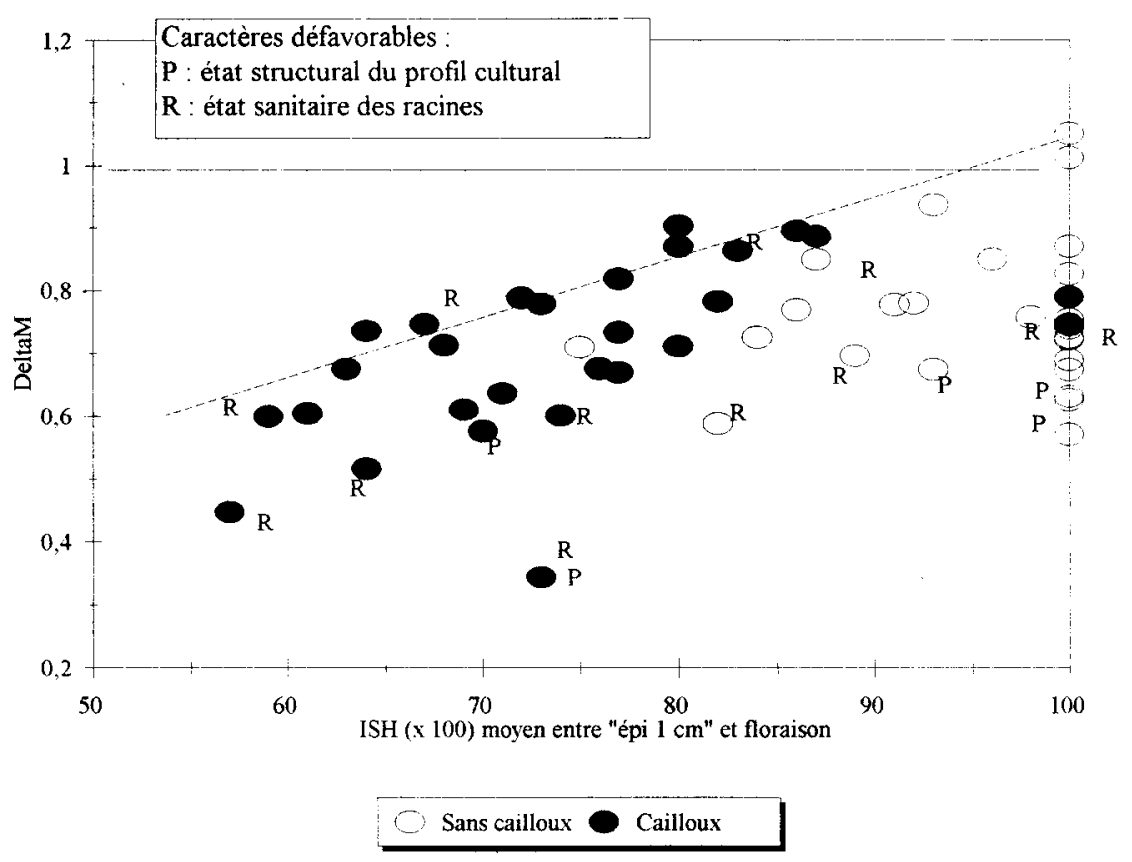

Fig 10. Relation entre indice de satisfaction (ISH) et croissance végétative pendant la montaison (Festival).
En appliquant les relations de référence entre biomasse végétative et nombre de grains déterminées à Grignon, on peut estimer que le nombre de grains potentiel varie de 13200 à 23100 pour Festival et de 13700 à 18700 pour Fidel. Sécheresse, état sanitaire des racines, état structural du profil jouent donc un rôle important sur la détermination du nombre de grains potentiel, par leur effet sur la biomasse élaborée entre le stade "épi $1 \mathrm{~cm}$ " et la floraison.

Comme pour le peuplement épi, la faculté de réaliser le nombre de grains potentiel n'est pas indépendante du niveau à atteindre. Les résultats sont présentés au tableau VII.

\section{Diagnostic sur la réalisation du nombre de grains $/ \mathrm{m}^{2}$ potentiel}

Nous avons ajouté les 3 points suivants aux éléments de diagnostic précédents :

i) l'alimentation azotée, exprimée par le rapport $(\beta)$ entre azote absorbé et nombre de grains, jugé par rapport à des valeurs de référence variétale. En accord avec la bibliographie (Meynard, 1989), nous avons considéré une valeur seuil de $0,9 \mathrm{~g}$ d'azote par millier de grains pour Festival et de 1,10 pour Fidel, en deçà de laquelle on conclut à une limite du fonctionnement par manque d'azote ;

ii) I'alimentation hydrique dans la décade de la floraison pour laquelle nous avons considéré une valeur seuil de I'ISH de 0,75 , dictée par la distribution des valeurs ;

iii) la température minimale du jour estimé de la méiose, daté par rapport à la date de floraison à laquelle on retranche 180 (pour Festival) ou 200 degrés-jour (pour Fidel). Nous avons repéré les parcelles où elle était inférieure ou égale à $1^{\circ}$. Là aussi, ce seuil résulte de la distribution des températures relevées à ces dates : 27 parcelles présentent des valeurs $\leq 1^{\circ}$ et $26 \geq$ à $6^{\circ}$. Deux parcelles sont intermédiaires avec une température de $3^{\circ}$.

Le bas du tableau VII synthétise les effets des facteurs considérés. Le facteur majeur qui ressort est la température minimale à la méiose ou, rappelons-le, tout autre facteur qui lui soit lié, comme par exemple le rayonnement. Aucune parcelle ayant subi des températures minimales à la méiose inférieures ou égales à $1^{\circ}$ ne parvient à réaliser son potentiel de nombre de grains. À l'opposé, la fréquence de telles parcelles est importante (61\%) dans les situations à mauvaise réalisation du potentiel. $\mathrm{Ni}$ les conditions de nutrition azotée, ni les conditions hydriques à la floraison ne sont discriminantes.

On constate que les forts taux de réalisation du potentiel de NG ne sont atteints qu'en l'absence de situations thermiques défavorables à la méiose : it ne s'agit que de parcelles en terres non caillouteuses n'ayant pas été semées parmi les plus précoces. Ailleurs, les conditions climatiques lors de la méiose interviennent dans près de 2 cas sur 3.

\section{Conclusion}

Dans le contexte de l'année, les conditions de nutrition azotée ne sont pas déterminantes. C'est l'alimentation hydrique, en interaction avec l'état 
Tableau VII. Répartition en nombre de parcelles des modalités de réalisation du nombre de grains par $\mathrm{m}^{2}$.

\begin{tabular}{|c|c|c|c|}
\hline & \multicolumn{3}{|c|}{ Degré de réalisation du nombre de grains $/ \mathrm{m}^{2}$ potentiel } \\
\hline & $>95 \%$ & $85-95 \%$ & $<85 \%$ \\
\hline \multicolumn{4}{|l|}{ Niveau du nombre de grains potentiel } \\
\hline Fidel $:>15900 / \mathrm{m}^{2}$ & 0 & 8 & 9 \\
\hline Festival : > $19600 / \mathrm{m}^{2}$ & - & $(2-4-4)$ & $(1-5-2)$ \\
\hline Fidel : $14000-15900 / \mathrm{m}^{2}$ & 6 & 10 & 12 \\
\hline Festival : $17300-19600 / \mathrm{m}^{2}$ & $(1-0-3)$ & $(1-6-1)$ & $(1-8-6)$ \\
\hline Fidel : $<14000 / \mathrm{m}^{2}$ & 1 & 2 & 7 \\
\hline Festival : $<17300 / \mathrm{m}^{2}$ & $(1-0-0)$ & $(0-0-2)$ & $(4-4-3)$ \\
\hline \multicolumn{4}{|l|}{ \% de parcelles affecté par: } \\
\hline Déficit hydrique à floraison & 43 & 35 & 39 \\
\hline Conditions climatiques à la méiose & 0 & 50 & 61 \\
\hline Azote limitant & 29 & 15 & 21 \\
\hline
\end{tabular}

$(x-y-z): x:$ nombre de parcelles avec azote limitant, $y:$ nombre de parcelles avec température minimale à la méiose $\leq 1^{\circ}, z:$ nombre de parcelles avec ETR/ETM dans la décade de la floraison $<0,75$.

du profil cultural et celui des racines, qui a une influence prépondérante sur l'accumulation de biomasse durant la montaison et, par là, sur la détermination du nombre de grains potentiels. Les accidents climatiques à la méiose jouent un rôle déterminant pour la réalisation de ce potentiel.

\section{Passage du nombre de grains $/ \mathrm{m}^{2}$ au rendement}

\section{Influence du nombre de grains $/ \mathrm{m}^{2}$}

Comme on peut le voir au tableau III, aucune parcelle n'atteint le rendement potentiel tel que nous l'avons défini, c'est-à-dire fixé par le niveau de rayonnement incident cumulé entre floraison et maturité. Pour bon nombre de parcelles, le nombre de grains est insuffisant compte tenu du poids individuel de grains potentiellement atteignable. Les conditions de l'année imposaient donc un nombre potentiel de grains très élevé, ou légèrement plus faible mais qui aurait dû être impérativement réalisé. Plus le nombre potentiel de grains et/ou son degré de réalisation sont faibles, plus le nombre de grains est limitant. On rejoint ici la forte liaison notée plus haut entre rendement et nombre de grains.

\section{Remplissage des grains (D/4)}

Les variations de NG étant importantes, nous n'avons pas pu mettre directement en relation le
PM observé et les conditions de post-floraison. Comme indiqué plus haut (voir «Rendement réalisé»), nous avons calculé le manque de rendement lié uniquement au défaut de remplissage des grains en considérant le rapport DeltaR. Multiplié par 100, il varie dans l'absolu de 0 (rendement nul parce que le remplissage des grains est nul) à 100 (le remplissage des grains ne limite pas le rendement). Dans nos parcelles, il varie de 77 à 99 . Nous avons mis en regard de cet indice les éléments de diagnostic suivants :

i) le degré de satisfaction des besoins en eau dans la 2e moitié de la période de post-floraison ;

ii) l'état du profil cultural ;

iii) l'état sanitaire des racines après floraison;

iv) la verse (pourcentage de tiges couchées);

v) l'indice I d'attaque des tiges par les champignons après la floraison.

Le degré de satisfaction des besoins en eau dans la seconde moitié de la période de post-floraison est prédominant comme on peut le constater à la figure 11. Cet effet majeur apparaît aggravé par les éléments affectant l'état des capteurs aériens mais surtout souterrains. Nous avons indiqué sur la figure les cas où l'état sanitaire des racines $(R)$ présente les plus mauvais scores (états $c$ et $d$ ), où le profil (P) est limitant, où la verse $(\mathrm{V})$ affecte plus de $25 \%$ des tiges, et où les maladies cryptogamiques affectent plus de $50 \%$ des tiges. 
Considérée sous cet angle, la réussite de la phase de remplissage varie de près de $25 \%$. C'est loin d'être négligeable, même si le nombre de grains reste la composante la plus déterminante du rendement final.

\section{Relations avec le milieu et les systèmes de culture}

À l'issue de cette phase de diagnostic, on peut envisager d'aborder le quatrième principe d'analyse présenté en introduction. Cela consistera tout d'abord à mettre en évidence les relations entre terrain, précédent, dates de semis et occurrence de caractères défavorables. Ensuite, on analysera la relation entre rendements et diagnostics effectués pour chaque phase.

\section{Relations entre terrains, systèmes de culture et caractères défavorables du milieu}

Seules 2 parcelles ne présentent aucune caractéristique défavorable. Ailleurs les problèmes les plus fréquemment en cause sont les accidents à la méiose liés aux conditions climatiques, la sécheresse à la montaison et en post-floraison, l'état sanitaire des tiges et surtout des racines et l'état structural du profil. Les autres caractères sont secondaires et n'interviennent pas dans plus de $20 \%$ des cas. Le tableau VIII présente, en résumé, les relations entre terrains, précédent cultural et date de semis du blé, et fréquence d'intervention de ces caractéristiques défavorables.

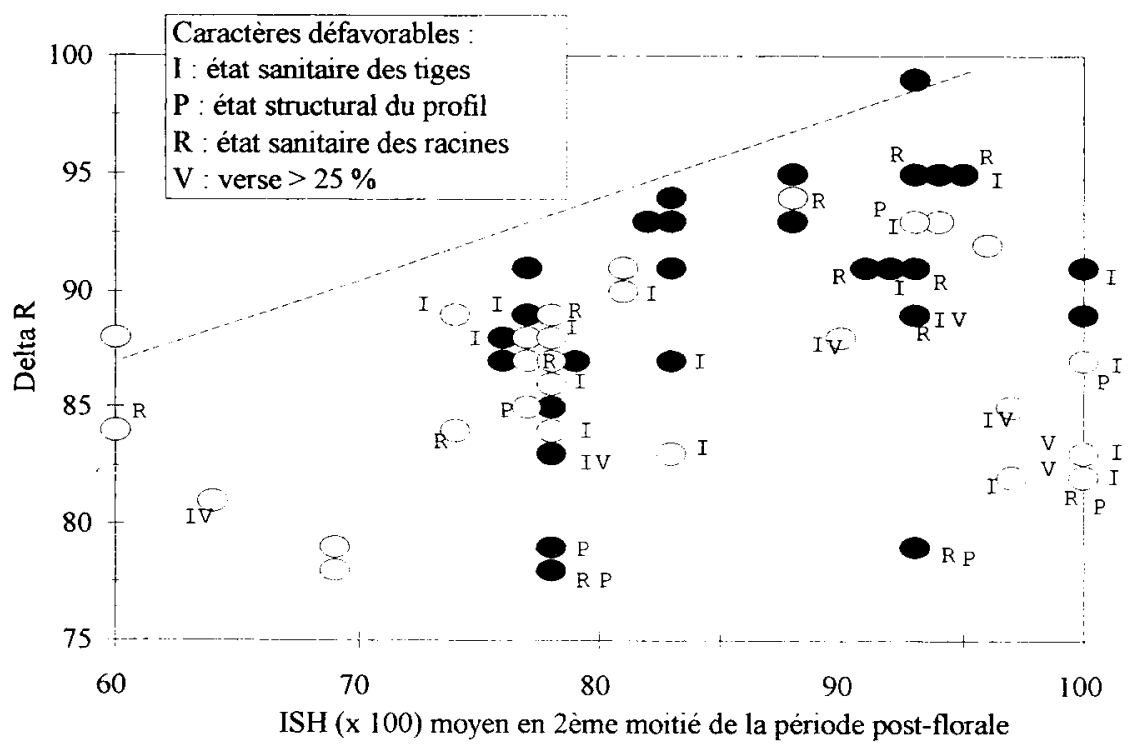

On constate que l'effet du terrain joue très fortement pour l'alimentation hydrique durant montaison et, en interaction avec la date de semis, pour les accidents à la méiose et l'alimentation hydrique post-floraison. II joue aussi pour l'état sanitaire des tiges où seules les terres sans cailloux sont concernées. L'effet du précédent cultural est surtout net pour l'état sanitaire des racines, d'autant plus qu'il s'agit de terres caillouteuses ou de semis tardifs en parcelles sans cailloux. L'effet de la date de semis n'est direct que pour l'état du profil cultural mais intervient de façon déterminante avec le terrain en interaction avec les événements climatiques du printemps et de l'été. On peut dire qu'en moyenne les précédents blé comportent plus de risques d'apparition de caractères défavorables que les précédents non-blé, ces risques étant renforcés par les semis tardifs.

\section{Relations entre diagnostics et rendements}

Le tableau IX présente, pour 4 classes de rendement, la fréquence observée d'absence de caractères défavorables pour chaque phase de diagnostic ainsi que la fréquence de semis tardifs, de terrains caillouteux et de précédents blé.

La fréquence des diagnostics sans caractère défavorable diminue lorsque le rendement diminue pour les 2 premières étapes. L'effet important du fonctionnement précoce sur le rendement apparait bien ici. Pour la $3^{e}$ étape, la fréquence élevée d'accidents à la méiose n'a pas conduit à de gros dysfonctionnements des peuplements. Lors de la $4^{\mathrm{e}}$ étape, sécheresse, état sanitaire 
Tableau VIII. Relation entre terrains, précédents, dates de semis et caractéristiques défavorables au blé.

\begin{tabular}{|c|c|c|c|c|c|c|c|c|}
\hline & \multicolumn{4}{|c|}{ Terres sans cailloux } & \multicolumn{4}{|c|}{ Terres avec cailloux } \\
\hline & \multicolumn{2}{|c|}{ Non-blé } & \multicolumn{2}{|c|}{ Blé } & \multicolumn{2}{|c|}{ Non-blé } & \multicolumn{2}{|c|}{ Blé } \\
\hline & $\begin{array}{r}\text { Semis } \\
<10 / 10\end{array}$ & $\begin{array}{r}\text { Semis } \\
\geq 10 / 10\end{array}$ & $\begin{array}{r}\text { Semis } \\
<10 / 10\end{array}$ & $\begin{array}{r}\text { Semis } \\
\geq 10 / 10\end{array}$ & $\begin{array}{r}\text { Semis } \\
<10 / 10\end{array}$ & $\begin{array}{r}\text { Semis } \\
\geq 10 / 10\end{array}$ & $\begin{aligned} & \text { Semis } \\
&<10 / 10\end{aligned}$ & $\begin{array}{r}\text { Semis } \\
\geq 10 / 10\end{array}$ \\
\hline $\begin{array}{l}\text { Accidents à la méiose } \\
\text { Sécheresse à la floraison } \\
\text { Sécheresse à la montaison } \\
\text { État sanitaire des tiges } \\
\text { État sanitaire des racines } \\
\text { État structural du profil }\end{array}$ & $\begin{array}{l}0 \\
0 \\
\bullet\end{array}$ & 0 & 0 & 0 & 0 & ? & 0 & 0 \\
\hline
\end{tabular}

Sans mention : effet absent ou très rare ; 0 : affecte occasionnellement les parcelles ; : affecte la majorité des parcelles du groupe ; - : affecte la quasi-totalité des parcelles.

dégradé et verse conduisent généralement à de faibles rendements. Les rendements les plus élevés se caractérisent plus par une dominance de facteurs et conditions favorables tout au long du cycle que par un type de parcelle. Dans tous les cas, il y a de «bonnes» parcelles. En revanche, les mauvais rendements, qui correspondent presque continuellement à la présence d'éléments défavorables, sont principalement le fait de semis tardifs en terres caillouteuses derrière blé.

En définitive, s'il n'existe pas de relation stricte entre les variables de structuration de ce réseau de parcelles et les rendements du blé, celles-ci semblent bien conduire à des niveaux de risque différenciés, pour l'extériorisation desquels, le climat, et donc la date de semis et la variété, jouent un rôle déterminant.

\section{CONCLUSION}

Dans ce diagnostic régional, nous avons accepté le risque de ne pas pouvoir effectuer de comparaisons simples de type expérimental entre les situations, afin de pouvoir couvrir une large gamme de variation représentative de la réalité. Pour surmonter cette difficulté, nous comptions utiliser d'une part une méthode éprouvée et d'autre part des références, notamment variétales, acquises ailleurs. Ce faisant, même si la méthode s'est avérée globalement performante, on a pu mettre en évidence certaines insuffisances qui amènent à nuancer les conclusions. Ainsi, les références variétales utilisées pour la phase d'installation du peuplement avant montaison sont très imparfaites. De même, l'évaluation du rendement potentiel à partir de la photosynthèse post-floraison pose problème du fait du

Tableau IX. Fréquence des diagnostics sans caractères défavorables selon la classe de rendement.

\begin{tabular}{|c|c|c|c|c|c|c|c|c|c|}
\hline \multirow{2}{*}{$\begin{array}{l}\text { Classe de } \\
\text { rendement } \\
\text { réalisé } \\
\text { (q/ha) }\end{array}$} & \multirow{2}{*}{$\begin{array}{c}\text { Amplitude } \\
\text { de réalisation } \\
\text { du potentiel } \\
\text { de rendement } \\
(r / p \times 100)\end{array}$} & \multirow[t]{2}{*}{$\begin{array}{l}\text { Nombre de } \\
\text { parcelles }\end{array}$} & \multicolumn{3}{|c|}{$\begin{array}{l}\text { Fréquence par classe } \\
\qquad(\%)\end{array}$} & \multicolumn{4}{|c|}{$\begin{array}{l}\text { Fréquence d'absence de } \\
\text { facteurs défavorables (\%) }\end{array}$} \\
\hline & & & $\begin{array}{c}\text { Terrain } \\
\text { avec cailloux }\end{array}$ & $\begin{array}{c}\text { Précédent } \\
\text { blé }\end{array}$ & $\begin{array}{l}\text { Semis } \\
\text { tardif }\end{array}$ & $D / 1$ & $D / 2$ & $D / 3$ & $D / 4$ \\
\hline$>75$ & $80-91$ & 18 & 56 & 50 & 44 & 78 & 61 & 33 & 56 \\
\hline $65-75$ & $70-83$ & 16 & 31 & 50 & 50 & 56 & 44 & 25 & 19 \\
\hline $55-65$ & $59-73$ & 14 & 43 & 36 & 77 & 36 & 50 & 14 & 28 \\
\hline$<55$ & $34-60$ & 7 & 100 & 86 & 86 & 0 & 14 & 28 & 14 \\
\hline
\end{tabular}


manque de précision du paramétrage (valeurs d'efficience d'interception, de conversion) et de la méconnaissance de certains mécanismes écophysiologiques (rôle des réserves, sénescence). II faut cependant noter que les observations de milieu sont cohérentes avec le diagnostic formulé à partir de ces références.

Cette expérience de diagnostic comporte des limites quant aux possibilités d'extrapolation : le fait qu'on n'ait étudié qu'une seule année climatique limite la portée des conclusions, notamment pour les mécanismes dont la connaissance trop imprécise empêche les possibilités de simulation qui permettraient de prévoir les effets des systèmes de culture sur le fonctionnement et les performances des peuplements cultivés. Cette étude contribue ainsi à mettre en évidence les besoins de recherche qui subsistent. On peut citer en premier lieu la nécessité d'approfondir les connaissances des lois régissant certains mécanismes, notamment le déterminisme du parasitisme et de ses conséquences, pour élaborer des modèles de prévision. En second lieu, l'application de ces modèles nécessitera des opérations de recherche pour :

- établir les références variétales nécessaires à la formulation des diagnostics ;

- établir les références climatiques fréquentielles, nécessaires pour que les connaissances évoquées ci-dessus soient réellement utilisables dans les schémas décisionnels des agriculteurs.

\section{RÉFÉRENCES}

Amir J, Sinclair TR (1991) A model of the temperature and solar-radiation effects on spring wheat growth and yield. Field Crop Res 28, 47-58

Boiffin J, Sebillotte M, Couvreur F (1976) Incidence de la simplification du travail sur l'élaboration des rendements du blé et du maïs. In : Simplification du travail du sol en production céréalière, ITCF, Paris, 239-280

Capillon A, Leterme P, Manichon H (1985) Les exploitations agricoles du Thymerais et leurs systèmes de culture. Une approche typologique. INRA-ONIC, 52 $p p+$ annexes

Catin G (1988) Analyse des composantes du rendement du blé tendre d'hiver. Mémoine Relance Agronomique, Ch Rég d'Agric du Centre, 70 pp + annexes

Gate $P$, Masse $J$ (1988) Blé récolte 1987, un bilan très contrasté. Persp Agric 121, 14-23

Gosse G, Varlet-Grancher C, Bohnomme R, Chartier $\mathrm{M}$, Allirand JM, Lemaire G (1986) Production maximale de matière sèche et rayonnement solaire intercepté par un couvert végétal. agronomie 6 (1), 47-56
Groot JJR, Spiertz JHJ (1990) Photosynthesis and nitrogen translocation in cereals during grain filling and implications for crop yield. Proc Int Congress Plant Physiol, New Delhi, 15-20/02/1988, vol 2, 1027-1033

Huet $P(1988)$ Notation de l'état sanitaire des racines et de la base des tiges du blé. Action concertée $\mathrm{RCl}$ INRA/ONIC/ITCF, $22 \mathrm{p}$ + annexes

INRA (1986) (ouvrage collectif) Les rotations céréalières intensives. Dix années d'études concertées INRA-ONIC-ITCF, 1973-1983. Publication INRA, $481 \mathrm{pp}$

Lamballe P, Sebill N (1986) Diagnostic sur les causes de variation des rendements réalisés dans le Boischaut nord de l'Indre. Mémoire DAA INA-PG

Leterme $P$ (1985) Modélisation de la croissance et de la production des siliques chez le colza d'hiver (Brassica napus L). Application à l'interprétation de résultats de rendement. $N^{\circ}$ Spécial Informations Techniques CETIOM, $112 \mathrm{p}$

Leterme P (1987a) Analyse des rendements réalisés par la variété Fidel dans les sites expérimentaux de Grignon, Le Rheu et Rouvroy-les-Merle en 1986. Action concertée RCI INRA/ONIC/ITCF, $24 \mathrm{pp}+$ figures et annexes

Leterme $P$ (1987b) Étude comparée du comportement de Fidel et Festival à Grignon. Action concertée RCl INRA/ONIC/ITCF, 14 pp

Maillet I (1992) Contribution à la modélisation du nombre d'inflorescences produites par le colza d'hiver (Brassica napus $L$ var oleifera). Thèse INA-PG, $186 \mathrm{pp}+$ annexes

Manichon H, Gautronneau Y (1987) Guide méthodique du profil cultural. GEARAVCEREF Lyon

Manichon H, Roger-Estrade J, Leterme P (1988) Analyse des profils culturaux et des itinéraires techniques. Action concertée RCI INRA/ONIC/ITCF, 22 $\mathrm{pp}+$ annexes

Masle J (1980) L'élaboration du nombre d'épis chez le blé d'hiver. Influence de différentes caractéristiques et de la structure du peuplement sur l'utilisation de l'azote et de la lumière. Thèse INA-PG, Paris, $274 \mathrm{pp}$

Masle J (1986) Méthodologie de l'analyse du rendement du blé d'hiver. Doc mult INRA-INA-PG, $30 \mathrm{pp}$ + annexes

Meynard JM (1985) Construction d'itinéraires techniques pour la conduite du blé d'hiver. Thèse INAPG, Paris, $297 \mathrm{pp}$

Meynard JM (1987) L'analyse de l'élaboration du rendement sur les essais de fertilisation azotée. Persp Agric 115, 76-83

Meynard JM (1989) Contribution à la réduction des risques de pollution des eaux par les nitrates d'origine agricole : conception d'itinéraires techniques de blé d'hiver. Doc mult INA-PG, 119 pp

Meynard JM, Sebillotte M (1994) L'élaboration du rendement du blé, base pour l'étude des autres céréales à paille. In : Le Point sur l'élaboration du 
rendement (D Picard, L Combe, eds), Éditions INRA, Paris, 31-51

Monteith JL (1972) Solar radiation and productivity in tropical ecosystems. J Appl Ecol 9, 747-766

Monteith JL (1994) Discussion - Validity of the correlation between intercepted radiation and biomass. Agric For Meteorol 68, 213-220

Navarro-Garza H (1984) L'analyse des composantes du rendement du maïs. Application à l'étude de la variabilité des rendements dans une petite région. Thèse DDI, INA-PG, $238 \mathrm{p}+$ annexes

Norman JM, Arkebauer TJ (1991) Predicting canopy light use efficiency from leaf characteristics. In : Modeling Plant and Soil Systems (JT Ritchie, J Harks, eds), Agronomy Monograph $n^{\circ} 31$, American Society of Agronomy, Madison, WI, États-Unis, 125143

O'Leary J, Connor DJ, White DH (1985) A simulation model of the development, growth and yield of wheat crop. Agric Syst 17, 1-26
Pluchard P (1989) Relations entre production de matière sèche de différentes variétés de blé et leurs composantes du rendement en grain. In : Colloque écophysiologie du blé, Versailles, Éditions INRA, Paris, 25-26/05/89

Rivoal R, Marzin H (1988) Analyses nématologiques. Action concertée RCI INRA/ONIC/ITCF, $9 p+$ annexes

Sebillotte M (1980) Analyse de l'élaboration du rendement de blé : un moyen pour résoudre les problèmes de cette culture. In: Wheat, technical monography, CIBA-GEIGY, Bâle, Suisse, 25-32

Szeicz G (1974) Solar radiation in plant canopy. J App/ Ecol 11, 1117-1156

Taureau JC (1987) Évaluation de l'offre en azote du milieu. Persp Agric 115, 38-45

Thorne GN, Pearman I, Day W, Todd AD (1988) Estimation of radiation interception by winter wheat from measurements of leaf area. J Agric Sci Camb $110,101-108$ 